\title{
The large $\mathrm{D}$ black hole membrane paradigm at first subleading order
}

\author{
Yogesh Dandekar, ${ }^{a}$ Anandita De, ${ }^{b}$ Subhajit Mazumdar, ${ }^{a}$ Shiraz Minwalla ${ }^{a}$ and \\ Arunabha Saha ${ }^{a}$ \\ ${ }^{a}$ Department of Theoretical Physics, Tata Institute of Fundamental Research, \\ Homi Bhabha Road, Mumbai 400005, India \\ ${ }^{b}$ Department of Physics, Indian Institute of Science Education and Research, \\ Dr. Homi Bhabha Road, Pashan, Pune 411008, India \\ E-mail: yogesh@theory.tifr.res.in, anu.anandita@gmail.com, \\ subhajitmazumdar@theory.tifr.res.in, minwalla@theory.tifr.res.in, \\ arunabha@theory.tifr.res.in
}

ABSTRACT: In the large D limit, and under certain circumstances, it has recently been demonstrated that black hole dynamics in asymptotically flat spacetime reduces to the dynamics of a non gravitational membrane propagating in flat $D$ dimensional spacetime. We demonstrate that this correspondence extends to all orders in a $1 / D$ expansion and outline a systematic method for deriving the corrected membrane equation in a power series expansion in $1 / D$. As an illustration of our method we determine the first subleading corrections to the membrane equations of motion. A qualitatively new effect at this order is that the divergence of the membrane velocity is nonzero and proportional to the square of the shear tensor reminiscent of the entropy current of hydrodynamics. As a test, we use our modified membrane equations to compute the corrections to frequencies of light quasinormal modes about the Schwarzschild black hole and find a perfect match with earlier computations performed directly in the gravitational bulk.

KEYwords: Black Holes, Classical Theories of Gravity

ARXIV EPRINT: 1607.06475 


\section{Contents}

1 Introduction $\quad 1$

1.1 Review of earlier work 1

1.2 The membrane paradigm at higher orders in $1 / D$

2 Perturbation theory: general structure $\quad \mathbf{5}$

2.1 A more detailed description of the starting ansatz 5

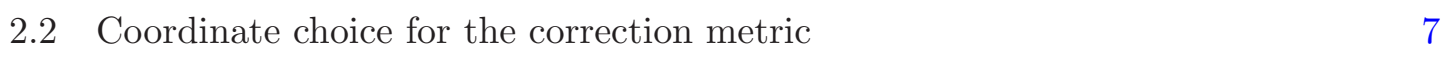

2.3 Orders of $D \quad 8$

2.4 All orders definition of the membrane surface and velocity 9

$\begin{array}{ll}2.5 & \text { Structure of the equations of perturbation theory } \\ \end{array}$

$\begin{array}{ll}2.6 & \text { The Einstein constraint equations } \\ & 11\end{array}$

$\begin{array}{ll}2.7 & \text { Choice of basis for the constraint and dynamical equations }\end{array}$

3 Perturbation theory at first order $\quad \mathbf{1 5}$

$\begin{array}{lll}3.1 & \text { Listing first order source functions } & 15\end{array}$

$\begin{array}{ll}3.2 \text { Tensor sector } & 16\end{array}$

$\begin{array}{lll}3.3 & \text { Vector sector } & 17\end{array}$

$\begin{array}{lll}\text { 3.3.1 Constraint equation and the membrane equation of motion } & 17\end{array}$

$\begin{array}{ll}\text { 3.3.2 Solving for the vector fluctuation } & 18\end{array}$

$\begin{array}{ll}3.4 \text { Scalar sector } & 19\end{array}$

$\begin{array}{lll}3.4 .1 & \text { Constraint equations and } \nabla . u & 19\end{array}$

3.4.2 Solving for the scalar fluctuations 20

$\begin{array}{ll}3.5 & \text { Final result for the first order metric } 22\end{array}$

4 2nd order solution $\quad 22$

4.1 Constraints on membrane data 23

4.1.1 Correction to the membrane equations from the vector sector 23

4.1.2 Divergence of velocity from a scalar constraint 25

$\begin{array}{ll}4.2 & \text { Second order corrections to the metric } 26\end{array}$

$\begin{array}{lll}4.2 .1 & \text { Tensor sector } & 26\end{array}$

$\begin{array}{lll}4.2 .2 & \text { Vector sector } & 26\end{array}$

$\begin{array}{lll}4.2 .3 & \text { Scalar sector } & 26\end{array}$

5 The spectrum of small fluctuations around a spherical membrane $\quad 27$

$\begin{array}{ll}5.1 \text { The divergence condition } & 27\end{array}$

5.2 Linearized equation of motion 28

$\begin{array}{lll}5.3 & \text { Scalar quasinormal modes } & 29\end{array}$

$\begin{array}{ll}5.4 \text { Vector quasinormal modes } & 29\end{array}$

6 Discussion $\quad 30$ 


\section{Introduction}

It has recently been noted that the classical dynamics of black holes simplifies in the limit of a large number of dimensions. The key observation - first made by Emparan, Suzuki, Tanabe and collaborators in [1-7] — is that black holes at large $D$ have two effective length scales. The first of these, $r_{0}$, is the size of the black holes. The second is the thickness of the black hole's gravitational tail, i.e. the distance beyond the black hole event horizon after which the gravitational potential rapidly decays to zero. In four dimensions the black hole size and thickness are comparable. In the large $D$ limit, however, the thickness of the gravitational tail turns out to scale like $r_{0} / D$ [1] and so is much smaller than the the black hole size.

This observation suggests the possibility of an effective 'dimensional reduction' of black hole dynamics to the membrane region; a slab of spacetime of thickness $1 / D$ centered around the codimension one event horizon. In work done over the last year, this expectation has been borne out in various contexts. In this paper we will focus on black holes propagating in an otherwise unperturbed flat space. Assuming that $r_{0}$ (see above) and the length scale of variation along the horizon are both of order unity, the dimensional reduction described above was worked out to leading nontrivial order in the $1 / D$ expansion for the most general nonlinear dynamical context in $[8,9]$; the special case of stationary solutions and their small fluctuations has also been studied at higher orders in the $1 / D$ expansion in [10-13]. In addition the dimensional reduction of small horizon ripples at length scale $1 / \sqrt{D}$ about particular solutions (black strings or black branes in flat, AdS or $d S$ space) has been studied in [14-18]. Further developments were presented in [19-25].

In this paper we further develop the general nonlinear dynamical construction of $[8,9]$. In particular we demonstrate that the reduction of black hole dynamics to membrane dynamics, worked out to leading nontrivial order in the $1 / D$ expansion in $[8,9]$, can be systematically generalized to every order in $1 / D$. As an application of this systematic framework we explicitly work out the first subleading corrections to the membrane equations of motion in the $1 / D$ expansion, and also determine the spacetimes dual to any particular membrane solution at next subleading order in the $1 / D$ expansion. In this introduction we first review the leading order construction presented in $[8,9]$ and then present our explicit higher order results.

\subsection{Review of earlier work}

Consider a class of $D$ dimensional metrics of the form

$$
g_{M N}=\eta_{M N}+\frac{\left(n_{M}-u_{M}\right)\left(n_{N}-u_{N}\right)}{\psi^{D-3}}
$$


The metrics (1.1) are parameterized by a smooth $D$ dimensional function $\psi$ and a smooth oneform field $u_{M} . n_{M}$ in (1.1) is the normal field to surfaces of constant $\psi$, (i.e. $n_{M}=$ $\frac{\partial_{M} \psi}{\sqrt{\partial_{P} \psi \partial_{Q} \psi \eta^{P Q}}}$ ). The oneform field $u_{M}$ is assumed to be unit normalized (i.e. $u_{N} u_{M} \eta^{M N}=$ -1 ) and tangent to surfaces of constant $\psi$ (i.e. $u_{M} n_{N} \eta^{M N}=0$ ).

In order to gain intuition for spacetimes of the form (1.1) it is useful to first consider a special case. Working with coordinates in which the metric on Minkowski space takes the form

$$
d s^{2}=-d t^{2}+d r^{2}+r^{2} d \Omega_{D-2}^{2},
$$

the choice $u=-d t$ and $\psi=\frac{r}{r_{0}}$ turns (1.1) into the metric of a Schwarzschild black hole of radius $r_{0}$ in the so called Kerr Schild coordinates.

Note $\psi=1$ is the event horizon of the Schwarzschild black hole. More generally the surface $\psi=1$ is easily verified to be a null submanifold of (1.1) for every choice of $\psi$ and $u$. This null manifold coincides with the event horizon of the (1.1) provided that $\psi$ and $u$ are chosen such that the metric (1.1) settles down into a collection of stationary black holes at late times. Following $[8,9]$ we refer to the submanifold $\psi=1$ as the membrane world volume. ${ }^{1}$

Note that as $\psi$ increases past unity $\frac{1}{\psi^{D-3}}$ decays to zero very rapidly. This decay is exponential in $D$ once $\psi-1 \gg \frac{1}{D}$. It follows that (1.1) represents a class of asymptotically flat spacetimes with the following property; the spacetime outside the event horizon deviates significantly from flat space only in a slab of thickness $\frac{1}{D}$ around the event horizon. We will refer to this as the membrane region. $[8,9]$ set out to characterize solutions of the vacuum Einstein equations, $R_{M N}=0$, that reduce to metrics of the form (1.1) in the large $D$ limit, with corrections in a power series in $\frac{1}{D}$. As we have reviewed above, when $\psi-1 \gg \frac{1}{D}$ the spacetimes (1.1) reduce to flat space. Deviations from flatness are nonperturbatively small in the $\frac{1}{D}$ expansion. Thus Einstein's equations are automatically solved at all order in $1 / D$ outside the membrane region. In order to obtain a true solution of Einstein's equations, the solution (1.1) needs to be corrected order by order in the $\frac{1}{D}$ expansion only in the membrane region.

Consider a region of size $\frac{1}{D}$ centered around any point $x_{0}$ on the event horizon of (1.1). It may be shown that the metric of this ball is closely approximated by the metric in an equivalent small region centered around the appropriate event horizon point of some boosted Schwarzschild black hole provided that

$$
\nabla^{2}\left(\frac{1}{\psi^{D-3}}\right)=0, \quad \nabla \cdot u=0
$$

(the contraction of all indices is achieved by use of the metric $\eta_{M N}$ in the equations above). ${ }^{2}$

\footnotetext{
${ }^{1}$ Through this paper we assume that $\psi$ in $(1.1)$ is chosen to ensure that the membrane surface is a smooth codimension one surface that is timelike when viewed as a submanifold of flat space (we have emphasized above that this surface is a null submanifold of the metric (1.1)). We also assume that $\psi$ is chosen to ensure that $\frac{1}{\psi^{D-3}}$ decays at spatial infinity.

${ }^{2}$ When an expression like $\nabla^{2}$ acts on $\frac{1}{\psi^{D-3}}$ we get two distinct terms of order $D^{2}$ in two ways. The first term is $\propto(D-3)(D-2) \frac{(\nabla \psi)^{2}}{\psi^{D-1}}$. The second term is $\propto(D-3) \frac{\nabla^{2} \psi}{\psi^{D-2}}$. Though the second term has one less explicit factor of $D$ than the first, it actually contributes at the same order in the $1 / D$ expansion - i.e. at
} 
These equations need only be satisfied at leading order in $D$ and can be violated at subleading orders. As Schwarzschild black holes are exact solutions to Einstein's equations, it follows as a consequence that the spacetimes (1.1) almost solve Einstein's equations in the membrane region, provided that (1.2) is satisfied at every point on the membrane.

The statement that Einstein's equations are 'almost' solved in the membrane region has the following precise meaning. When evaluated in the membrane region the four derivative scalar $R_{A B} R^{A B}$ is in general of order $D^{4}$. This estimate follows immediately from the fact that the metric varies on a length scale of order $1 / D$ in the membrane region. Once we impose (1.2), on the other hand, $R_{A B} R^{A B}$ turns out to be of order $D^{2}$, i.e. In a coordinate system in which all components of the metric are of order unity, $R_{A B}$ is of order $D$; one order lower than the generic order suggested by a dimensional estimate. In other words (1.2) ensures that Einstein's equations are obeyed to leading order — but are generically violated at first subleading order. Consequently the metrics (1.1) - with the conditions (1.2) imposed at leading order- are plausible starting points for the construction of true solutions of Einstein's equations in a power series in $\frac{1}{D}$.

The authors of $[8,9]$ were able to carry out this perturbative expansion to first subleading order in $\frac{1}{D}$ (see below for a review). Interestingly they discovered that arbitrary metrics of the form (1.1) could not be corrected to yield regular solutions to Einstein's equations at next order in $\frac{1}{D}$. It turns out to be possible to correct (1.1) at first order in $1 / D$ only when the fields $\psi$ and $u$ obey an integrability constraint - a membrane equation of motion - that we will describe in considerable detail below. Whenever this condition is obeyed, a regular correction (of order $1 / D$ ) to the metric (1.1) was found in $[8,9]$. The corrected metric obeys $R_{A B}=\mathcal{O}(1) ;^{3}$ i.e. once the corrections are taken into account, Einstein's equations are solved at leading and first subleading order in $\frac{1}{D}$.

We now turn to a description of the integrability constraints mentioned in the previous paragraph. Consider the surface $\psi=1$, viewed as a submanifold of flat space with metric $\eta_{M N}$; we refer to this submanifold as the membrane. Let $K_{M N}$ represent the extrinsic curvature of this (generically timelike) submanifold. Recall also that the velocity oneform field $u_{M}$ on the membrane surface is tangent to the membrane and so may be regarded as a oneform field in the membrane world volume. The authors of $[8,9]$ found that the metric (1.1) could be corrected to a regular ${ }^{4}$ solution of Einsteins equations at first order

\footnotetext{
leading order - because of the contraction of indices in $\nabla^{2}$. This is the reason that (1.1) solves the leading order equations only if $\nabla^{2} \psi$ takes the same value as it does in a Schwarzschild black hole, leading to the first requirement listed in (1.2). In a similar manner worldvolume derivatives of the horizon shape and velocity field - which are of order unity - compete with derivatives acting on $\frac{1}{\psi^{D}-3}$ only if their order is enhanced by the contraction of a worldvolume index. The only first derivative expression involving the black hole velocity that has such a contraction is $\nabla . u$. It follows that (1.1) satisfies the leading order equations only if $\nabla . u$ takes the same value as it does on a Schwarzschild black hole. This leads to the second of (1.2).

${ }^{3}$ More precisely, $R_{A B}=\mathcal{O}(1)$ in coordinates in which all metric components are of order unity. More generally, $R_{A B} R^{A B}$ is of order unity.

${ }^{4} \mathrm{By}$ a regular solution we mean a solution with a smooth event horizon that is regular everywhere outside the event horizon.
} 
if and only if the following constraints are obeyed

$$
\left(\frac{\nabla^{2} u_{A}}{\mathcal{K}}-\frac{\nabla_{A} \mathcal{K}}{\mathcal{K}}+u_{C} K_{A}^{C}-u . \nabla u_{A}\right) \mathcal{P}_{B}^{A}=0
$$

where $\mathcal{P}_{B}^{A}=\delta_{B}^{A}+u^{A} u_{B}$ is the projector orthogonal to the velocity vector on the membrane world volume, and all covariant derivatives are taken with respect to the induced metric on the membrane. The quantity $\mathcal{K}$ is the trace of the extrinsic curvature of the membrane worldvolume.

The integrability conditions (1.3) have an interesting interpretation. They may be thought of as a set of $D-2$ equations for $D-2$ variables (one of these variables is the shape of the membrane, and the other $D-3$ variables are the components of the unit normalized, divergence free velocity field). In other words the equations (1.3) define an initial value problem for membrane dynamics. As every configuration that obeys (1.3) gives rise to a metric that obeys Einstein's equations to the appropriate order in $1 / D$, it follows that solutions of the membrane equations (1.3) are in one to one correspondence with asymptotically flat dynamical black hole configurations that solve Einstein's equations to first subleading order in $1 / D$.

\subsection{The membrane paradigm at higher orders in $1 / D$}

In this paper we demonstrate that first order perturbative procedure outlined above extends systematically to arbitrary orders in the expansion in $\frac{1}{D}$. We will now very briefly outline our inductive argument. We assume that the perturbative procedure has been implemented upto $n^{\text {th }}$ order, i.e. that corrections to the metric (1.1) have been determined upto $n^{\text {th }}$ order in the $1 / D$ expansion in such a manner that $R_{M N}$ evaluated on the corrected solution is of order $D^{1-n}$. We then add further corrections of order $1 / D^{n+1}$ to the metric (see (2.7) and (2.10)). At order $D^{n-1}$ we demonstrate that the Einstein constraint equations are independent of the new unknown correction functions when evaluated on the event horizon $\psi=1$. These equations determine the correction to the membrane equations (and the divergence condition on the velocity) at order $1 / D^{n+1}$. Moving away from the horizon we argue that the order $D^{1-n}$ part of $R_{M N}$ takes the form listed in table 2. Setting the expressions in this table yields a set of inhomogeneous linear differential equations that can be used to determine order $1 / D^{n+1}$ corrections to the metric. Explicit expressions for the sources in these differential equations can only be obtained by grinding through the perturbative procedure, but we use a contracted Bianchi identity to demonstrate that the sources that occur in these equations are not all independent, but obey certain relations (see (2.25)) at every order of perturbation theory. Using these relations we are able to integrate the inhomogeneous differential equations for any source functions and obtain an explicit and unique expressions for the metric corrections at order $1 / D^{n+1}$ (see section 3 ) that are manifestly regular and obey all required boundary conditions.

As an illustration of the general method outlined above we explicitly implement the perturbative procedure to second subleading order in $\frac{1}{D}$. We find that the modified mem- 
brane equations take the form

$$
\begin{aligned}
& {\left[\frac{\nabla^{2} u_{A}}{\mathcal{K}}-\frac{\nabla_{A} \mathcal{K}}{\mathcal{K}}+u^{B} K_{B A}-u \cdot \nabla u_{A}\right] \mathcal{P}_{C}^{A}} \\
& +\left[\left(-\frac{u^{C} K_{C B} K_{A}^{B}}{\mathcal{K}}\right)+\left(\frac{\nabla^{2} \nabla^{2} u_{A}}{\mathcal{K}^{3}}-\frac{u \cdot \nabla \mathcal{K} \nabla_{A} \mathcal{K}}{\mathcal{K}^{3}}-\frac{\nabla^{B} \mathcal{K} \nabla_{B} u_{A}}{\mathcal{K}^{2}}-2 \frac{K^{C D} \nabla_{C} \nabla_{D} u_{A}}{\mathcal{K}^{2}}\right)\right. \\
& +\left(-\frac{\nabla_{A} \nabla^{2} \mathcal{K}}{\mathcal{K}^{3}}+\frac{\nabla_{A}\left(K_{B C} K^{B C} \mathcal{K}\right)}{\mathcal{K}^{3}}\right)+3 \frac{(u \cdot K \cdot u)\left(u \cdot \nabla u_{A}\right)}{\mathcal{K}}-3 \frac{(u \cdot K \cdot u)\left(u^{B} K_{B A}\right)}{\mathcal{K}} \\
& \left.-6 \frac{(u \cdot \nabla \mathcal{K})\left(u \cdot \nabla u_{A}\right)}{\mathcal{K}^{2}}+6 \frac{(u \cdot \nabla \mathcal{K})\left(u^{B} K_{B A}\right)}{\mathcal{K}^{2}}+\frac{3}{(D-3)} u \cdot \nabla u_{A}-\frac{3}{(D-3)} u^{B} K_{B A}\right] \mathcal{P}_{C}^{A}=0
\end{aligned}
$$

while the divergence free condition on the velocity field is modified, at second subleading order, to the equation

$$
\nabla \cdot u=\frac{1}{2 \mathcal{K}}\left(\nabla_{(A} u_{B)} \nabla_{(C} u_{D)} \mathcal{P}^{B C} \mathcal{P}^{A D}\right)
$$

Note that the first line in (1.4) is simply a rewriting of (1.3); the 2nd-4th lines of this equations represent corrections to (1.3). There is a well defined sense (see below) in which each of these correction terms is of order $\frac{1}{D}$ relative to the leading order terms in the first line. It follows that the equations (1.4) represent small corrections to the leading order equations (1.3). The first order corrected membrane equation of motion (1.4) and (1.5) are the main result of this paper.

We then present explicit expressions for the second order sources for all the inhomogeneous differential equations (see table 6). Plugging these sources into the general equations for the metric corrections at any order we obtain explicit results for the second order correction to the spacetime metric dual to any particular solution of the membrane equations of motion.

The second order corrected membrane equations (1.4) admit a simple solution; a spherical membrane at rest. This solution is dual to the Schwarzschild black hole. As a check of our second order corrections to the membrane equations we use (1.4) to compute the spectrum of small fluctuations about this simple solutions. This spectrum is easy to obtain, and turns out to be in perfect agreement with the second order corrected spectrum of quasinormal modes obtained by Emparan Suzuki and Tanabe in [6], providing confidence in the correctness of (1.4).

\section{Perturbation theory: general structure}

\subsection{A more detailed description of the starting ansatz}

As we have explained in the introduction, the starting point of our perturbative construction of large $D$ solutions to Einstein's equations is the metric (1.1). In the introduction we noted that the metrics (1.1) are parameterized by the $D$ dimensional function $\psi$ and the oneform field $u$. We assume these fields have a good large $D$ limit, i.e. that the length scale 
of variation in $\psi$ and $u$ is of order unity. Following $[8,9]$, however, consider two different functions $\psi$ with the same membrane surface (i.e. with coincident zero sets for $\psi-1$ ). These two functions define metrics (1.1) that coincide (outside the event horizon) at leading order in $1 / D$ but differ at subleading orders in $1 / D$. Similarly $u$ functions that agree on the membrane but differ off it lead to metrics (1.1) that differ only at subleading order in $1 / D$.

Any two metrics (1.1) that differ only at subleading orders in $1 / D$ constitute equivalent starting points for the perturbative construction of solutions in the following sense: the end result of perturbation theory starting from the two different starting points will be the same. In order to construct all distinct final metrics we need only consider one member of each 'equivalence class' of metrics (1.1). As explained above the equivalence classes are labeled by the zero set of the function $\psi-1$ (the membrane world volume) and the value of the velocity field on the membrane world volume. In order to pick a representative from each equivalence class that we can use to set up our perturbation theory we invent an arbitrary way of constructing the full function $\psi$ from its zero set, and the full velocity field $u$ from its values on the membrane. Following $[8,9]$ we refer to the (essentially arbitrary) rule for achieving this construction as a subsidiary condition on the functions $\psi$ and $u$.

For technical reasons, in this paper we utilize the subsidiary conditions of [8] rather than that of [9]. We now describe these conditions in detail.

Consider a given timelike membrane submanifold in flat space. At each point on the manifold consider a geodesic that shoots outwards from the manifold along its normal vector. The resultant collection of curves $^{5}$ is a spacefilling congruence of spacelike geodesics; caustics of this congruence, if any, only occur at distances of order unity (rather than $1 / D$ ) away from the membrane. ${ }^{6}$ We define the scalar function $B$ in the neighborhood of the membrane as follows; $B$ at any point is defined to be the signed proper distance, along the geodesic that passes through it, to the membrane. This distance is defined to be positive outside the membrane and negative inside the membrane. Note that $B$ vanishes on the membrane. We define

$$
n_{M}=\nabla_{M} B
$$

It follows from our construction above that

$$
n . n=1
$$

$n_{A}$ is the normal oneform to surfaces of constant $B$. We use the symbol $K_{M N}$ denote the extrinsic curvature of surfaces of constant $B$. Note of course that $n^{A} K_{A B}=0$. We also define $\mathcal{K}=K_{A}^{A}$. We then proceed to define the function $\psi$ as

$$
\psi=1+\frac{\mathcal{K} B}{D-3}
$$

\footnotetext{
${ }^{5}$ These 'curves' are actually straight lines as they are all geodesics in flat space. We use the term 'curve' to bring to mind the obvious generalization of this construction when the membrane is embedded in a curved spacetime.

${ }^{6}$ The quantity $\frac{D}{\mathcal{K}}$ gives a rough estimate for the distance away from the membrane at which the geodesics caustic. Below we explain that $\mathcal{K}$ is of order $D$ so that this caustic length scale is of order unity.
} 
In a similar manner we use the velocity function on the membrane to define a velocity oneform field in spacetime simply by parallel transport along our congruence of geodesics. It follows from our definitions above that

$$
\begin{aligned}
& n . \nabla n_{A}=0 \\
& n . \nabla u_{A}=0
\end{aligned}
$$

The first line of (2.4) follows upon differentiating 0 (2.2), using (2.1) and interchanging derivatives. This equation is in fact simply the geodesic equations for the congruence of geodesics that defines $B$. The equation on the second line of (2.4) follows from the fact that $u$ is defined off the membrane by parallel transport. It follows from (2.4) that

$$
K_{A B}=\left(\eta_{A}^{C}-n_{A} n^{C}\right)\left(\nabla_{C} n_{D}\right)\left(\eta_{B}^{D}-n^{D} n_{B}\right)=\left(\nabla_{A}-n_{A}(n . \nabla)\right) n_{B}=\nabla_{A} n_{B}=\nabla_{A} \nabla_{B} B
$$

Note that our definition of $n_{A}$ in this section, and the rest of this paper, differs slightly from the definition given in the introduction. The two definitions agree at leading order (which was all that was required in the discussion around (1.1) ) but differ at subleading orders in $1 / D$. The vector $n_{A}$ defined in this section - rather than the normal vector defined in the introduction - will be used through the rest of this paper.

Using (2.3) it is easily verified that on the submanifold $B=0$

$$
\begin{aligned}
\psi \nabla^{2} \psi & =\frac{\mathcal{K}^{2}}{D-3}+2 \frac{n \cdot \nabla \mathcal{K}}{D-3} \\
(D-2) \nabla \psi \cdot \nabla \psi & =\frac{D-2}{D-3} \frac{\mathcal{K}^{2}}{D-3}
\end{aligned}
$$

As we explain below, in the large $D$ limit taken in this paper $2 \frac{n . \nabla \mathcal{K}}{D-3}$ is of order unity while $\frac{\mathcal{K}^{2}}{D-3}$ is order $D$. It follows that to leading order in $D$

$$
(D-2) \nabla \psi \cdot \nabla \psi=\psi \nabla^{2} \psi, \quad \text { i.e. } \nabla^{2}\left(\frac{1}{\psi^{D-3}}\right)=0
$$

In other words our construction satisfies the first equation of (1.2). We satisfy the second equation in (1.2) by construction; we simply choose our $u$ oneform on the membrane such that its divergence vanishes at leading order in $D$. The divergence of $u$ will turn out not to vanish at a subleading order.

\subsection{Coordinate choice for the correction metric}

In this paper we search for solutions of Einstein's equations in a power series expansion in $\frac{1}{D}$

$$
\begin{aligned}
G_{M N} & =\eta_{M N}+h_{M N}, \\
h_{M N} & =\sum_{n=0}^{\infty} \frac{h_{M N}^{(n)}}{(D-3)^{n}}, \\
\text { with, } h_{M N}^{(0)} & =\frac{O_{M} O_{N}}{\psi^{D-3}},
\end{aligned}
$$


Here

$$
O_{M}=n_{M}-u_{M}
$$

We fix coordinate redefinition ambiguities by demanding

$$
h_{M N} O^{N}=0,
$$

Consider any point in the metric (1.1). The tangent space built about this point has two special vectors; the vector $n$ and the vector $u$. All the other $D-2$ directions orthogonal to $n$ and $u$ are equivalent and can be rotated into each other. It is thus useful to parameterize the most general fluctuation field $h_{M N}$ (subject to the gauge condition (2.9)) in the form

$$
h_{M N}^{(n)}=H^{(S, n)} O_{M} O_{N}+O_{(M} H_{N)}^{(V, n)}+H_{M N}^{(T, n)}+\frac{1}{D-3} H^{(T r, n)} \mathcal{P}_{M N},
$$

where,

$$
\begin{aligned}
\mathcal{P}_{M N} & =\eta_{M N}-O_{M} n_{N}-O_{N} n_{M}+O_{M} O_{N}, \\
O^{N} H_{N}^{(V, n)} & =0, \quad n^{N} H_{N}^{(V, n)}=0, \quad O^{M} H_{M N}^{(T, n)}=0, \quad n^{M} H_{M N}^{(T, n)}=0, \quad \mathcal{P}^{M N} H_{M N}^{(T, n)}=0,
\end{aligned}
$$

The superscripts $S, V$ and $T$ stand for scalar, vector and tensor respectively, and denote the transformation properties of the relevant symbol under the $\mathrm{SO}(D-2)$ rotations in tangent space that leave $n$ and $u$ fixed. The superscript $T r$ stands for trace, and labels a second scalar.

\section{$2.3 \quad$ Orders of $D$}

As we have explained above, in this paper we solve Einstein's equations in a systematic expansion in $\frac{1}{D}$. In order for this process to be well defined, we need to be able to unambiguously estimate the scaling with $D$ of various terms that appear in the metric and in the membrane equation of motion. Such an estimation is only unambiguous within subclasses of solutions, as we will now explain with an example.

Consider a membrane whose world volume is a $D-2$ sphere (of radius $R$ ) times time. The trace of extrinsic curvature, $\mathcal{K}$, of this surface is easily shown to be $\frac{D-2}{R}$ and so is of order $D$ (assuming $R$ is of order unity). On the other hand the surface $S^{p} \times R^{D-2-p}$ times time has $\mathcal{K}=\frac{p}{R}$. If $p$ and $R$ are both held fixed as $D$ is taken to infinity, $\mathcal{K}$ is of order unity for this surface. It follows that $\mathcal{K}$ cannot unambiguously be assigned a scaling with $D$ without making further assumptions. The same holds true of various other quantities (e.g. $\nabla^{2} u_{M}$ ) that enter the metric and equation of motion.

In this paper we follow $[8,9]$ and estimate the $D$ scalings of all terms as follows. We assume that

- Our starting ansatz is constructed by sewing together bits of the event horizon of black holes of radii $R$ and timelike velocity $u^{M}$ where $R$ and $u^{M}$ are everywhere finite and of order unity.

- Our starting configuration (and so our full solution) preserves an $\mathrm{SO}(D-p-2)$ rotational invariance with $p$ held fixed as $D$ is taken to infinity 
As explained in [9], these assumptions unambiguously specify the scaling with $D$ of all quantities of interest (in particular they force $\mathcal{K}$ to be of order $D$ ).

We emphasize that in this paper we use the assumptions listed above only to estimate the scalings of $D$ of various quantities. When the assumptions listed in the previous paragraph are obeyed, the membrane equations and metrics listed in this paper certainly apply. However the formulae of this paper apply more generally to any spacetime whose variables scale with $D$ in the same manner in which they would if the assumptions above were obeyed - a much larger class of configurations.

\subsection{All orders definition of the membrane surface and velocity}

As explained in subsection 2.1, the metric (1.1) - the starting point of our perturbative expansion - is completely determined by the shape of a membrane and a velocity field on the membrane. To what precision can this procedure be reversed? In other words if we are given a solution to Einstein's equations of the appropriate kind, how precisely can we read off the corresponding 'shape' and 'velocity' of the membrane?

We could attempt to identify the membrane shape and velocity field by simply expanding the exact solution in powers of $1 / D$ and focusing attention on the leading order term. By comparing with (1.1) we could then read off the membrane shape and velocity field. While this procedure is simple, a moment's thought will convince the reader that it is ambiguous at all orders in $1 / D$ save the leading order. ${ }^{7}$ In other words the requirement that our solution reduce to (1.1) defines the membrane shape and velocity only at leading order, leaving the subleading corrections to these quantities ambiguous. In this subsection we will fix this ambiguity by adopting a more precise definition of the shape and velocity field. This definition agrees with that of (1.1) at leading order, but is precise at all orders. We use this precise definition in the computations presented in the rest of this paper.

We define the membrane shape to be the location of the event horizon of our spacetime, and will choose higher order corrections to the metric (1.1) to ensure that this event horizon coincides with the surface $\psi=1$.

Turning to the velocity field, let $G^{A B}$ denote the full spacetime inverse metric. Let $n_{A}$ be the oneform normal to the event horizon. We define the velocity field on the membrane by the requirement that

$$
u^{A}=G^{A B} n_{B}
$$

on the event horizon (i.e. at $\psi=1$ ). In other words the velocity field is a tangent vector to the generators of the event horizon. It is easily verified that (2.11) is a true equation for the starting point of perturbation theory (1.1). We will choose corrections to the perturbative ansatz to ensure that (2.11) holds at all orders in $1 / D$.

The requirement (2.11) together with the requirement that $\psi=1$ is the exact event horizon of our spacetime are easily seen to be satisfied provided that

$$
\begin{aligned}
& H^{(S)}(\psi=1)=0 \\
& H_{M}^{(V)}(\psi=1)=0
\end{aligned}
$$

\footnotetext{
${ }^{7}$ For instance, the velocity redefinition $u^{\mu} \rightarrow u^{\mu}+\delta u^{\mu} / D$ does not change the metric at leading order in $1 / D$.
} 
The first condition ensures that $G^{M N} \partial_{M} \psi \partial_{N} \psi=0$, i.e. $d \psi$ is null at $\psi=1$ while the second condition then ensures that the full spacetime metric on the event horizon takes the form

$$
\eta_{M N}+O_{M} O_{N}+H_{M N}^{(T)}+\frac{1}{D-3} H^{T r} \mathcal{P}_{M N}
$$

Let us write this metric in a the local basis of oneforms $\left(n, u, Y_{a}\right)$ where $Y_{a}$ is any $D-2$ dimensional basis of oneforms chosen orthogonal to $n$ and $u$. In this basis the metric takes a block diagonal form with a $2 \times 2$ block (with basis $n$ and $u$ ) and a $D-2 \times D-2$ block (with basis $Y_{a}$ ). It follows that the inverse metric also has this block diagonal structure. Note that the $2 \times 2$ block is universal, i.e. it is the same at every order in perturbation theory. This block is the only one that contributes in (2.11). As (2.11) holds at leading order, it follows that the conditions (2.12) ensure that (2.11) holds at every order in perturbation theory.

Recall that according to (1.2) the velocity field used in (1.1) is divergence free at leading order in $\frac{1}{D}$. As we will see below, the divergence of the velocity field defined in this subsection will not, in general, vanish at subleading orders in $1 / D$.

\subsection{Structure of the equations of perturbation theory}

Our perturbative procedure proceeds as follows. We assume that our solution takes the form (2.7) together with (2.9) and (2.10). The Ricci tensor of this metric - evaluated in a slab of spacetime of thickness $1 / D$ around $\psi=1$ - takes the schematic form

$$
R_{M N}=\sum_{n} D^{2-n} R_{M N}^{n}
$$

Let us imagine that we have implemented our perturbative procedure to order $n$-1, i.e. that we have determined $h_{M N}^{(m)}$ for $m=1 \ldots n-1$ in a manner that ensures that $R_{M N}^{(m)}=0$ for $m=0 \ldots n-1$. In order to go to one higher order in perturbation theory we must solve for $h_{M N}^{(n)}$ to ensure that $R_{M N}^{n}$ also vanishes.

Schematically

$$
R_{M N}^{(n)}=C_{M N}^{P Q} h_{P Q}^{(n)}+\mathcal{S}_{M N}^{(n)}
$$

where $C_{M N}^{P Q}$ is a linear differential operator with derivatives only in the $\psi$ direction and $\mathcal{S}_{M N}^{(n)}$ is a source function. As $h_{P Q}^{(n)}$ is already of order $n$, the differential operator $C_{M N}^{P Q}$ is built entirely out of the zero order background metric (1.1), and so is the same at every order. On the other hand the source function $\mathcal{S}_{M N}^{(n)}$ is proportional to expressions of $n^{\text {th }}$ order in $1 / D$ built out of derivatives of the membrane velocity and shape function, and is different at every order.

At every point of the event horizon of the ansatz metric (1.1) there are two distinguished vectors; $n^{A}$ and $u^{A}$. Let

$$
\mathcal{P}_{A B}=\eta_{A B}-n_{A} n_{B}+u_{A} u_{B}
$$

denote the projector orthogonal to these two vectors (all dot products taken in flat space). Instead of dealing directly with the components of $R_{M N}$ we find it more convenient to use a basis adopted to $u^{A}$ and $n^{A}$ listed in table 1 . 


\begin{tabular}{|c|c|c|}
\hline Scalar sector & Vector sector & Tensor sector \\
\hline$R^{S_{1}}=O^{M} R_{M N} O^{N}$ & $R_{L}^{V_{1}}=O^{M} R_{M N} \mathcal{P}_{L}^{N}$ & $R_{A B}^{T}=\mathcal{P}_{A}^{M} R_{M N} P_{B}^{N}-\frac{\mathcal{P}_{A B}}{D-2} \mathcal{P}^{M N} R_{M N}$ \\
$R^{S_{2}}=O^{M} R_{M N} u^{N}$ & $R_{L}^{V_{2}}=u^{M} R_{M N} \mathcal{P}_{L}^{N}$ & \\
$R^{S_{3}}=u^{M} R_{M N} u^{N}$ & & \\
$R^{S_{4}}=R_{M N} \mathcal{P}^{M N}$ & & \\
\hline
\end{tabular}

Table 1. Basis of components of $R_{M N}$.

By explicit computation (plugging (2.7) into the formula for the Ricci tensor) we find that the linear combinations listed in table 1 of the curvature components $R_{M N}^{n}$ (see (2.13)) are given by the expressions listed in table 2 .

In table 2, fluctuation fields $H^{S}, H^{T r} H_{A}^{V}$ and $H_{M N}^{T}$ are taken to be of $n^{\text {th }}$ order and all source functions (e.g. $\mathcal{S}^{S_{1}}$ ) also understood to be $n^{\text {th }}$ order sources. All appearances of $\nabla \cdot u^{8}$ in the table 2 should also be understood as follows. Naively $\nabla . u$ is of order $D$. For that reason we expand

$$
\nabla \cdot u=(D-3)\left(\sum_{n=0}^{\infty} \frac{(\nabla \cdot u)_{n}}{(D-3)^{n}}\right)
$$

Every appearance of $\nabla . u$ in table 2 should actually be replaced by $(\nabla \cdot u)_{n}$. We have already seen in the introduction that $(\nabla \cdot u)_{0}=0$. We will see below that $(\nabla \cdot u)_{1}$ also vanishes, but that $(\nabla \cdot u)_{2}$ is nonzero.

In order to obtain table 2 we have worked in the neighbourhood of the surface $\psi=1$ and the variable $R$ is defined by $R=(D-3)(\psi-1) \cdot{ }^{9}$

\subsection{The Einstein constraint equations}

In the process of solving for the fluctuation fields $h_{M N}^{(n)}$ we will find the Einstein constraint equations (relevant to the foliation of our spacetime in slices of constant $\psi$ ) particularly useful. We will now provide a careful definition of these equations.

Let us define

$$
E_{M N} \equiv R_{M N}-\tilde{R} \frac{G_{M N}}{2}
$$

where $\tilde{R}$ is the Ricci scalar. The constraint equations are defined by the relations

$$
E_{M}^{(e c)}=E_{M N} G^{N L} n_{L}
$$

We have a total of $D$ constraint equations. These equations decompose into two scalars and one vector under local $\mathrm{SO}(D-2)$ rotations.

\footnotetext{
${ }^{8} \nabla . u$ is the divergence of the velocity field thought of as a vector field in $R^{D-1,1}$. On the surface $\psi=1$, however, $\nabla . u$ coincides with the membrane worldvolume divergence of velocity field (this follows upon using the second of (2.4)).

${ }^{9}$ We will explain below that the sources listed in table 2 are not completely independent, but are constrained by the well known relation

$$
\nabla^{M}\left(R_{M N}-\frac{\tilde{R}}{2} G_{M N}\right)=0 .
$$




\begin{tabular}{c} 
Scalar sector \\
\hline$R^{S_{1}}=\left(\frac{-\mathcal{K}^{2}}{2(D-3)^{2}}\right) \frac{d^{2} H^{(T r)}}{d R^{2}}+\mathcal{S}^{S_{1}}(R)$ \\
$R^{S_{2}}=\left(\frac{\mathcal{K}^{2}}{2(D-3)^{2}}\right) e^{-R} \frac{d}{d R}\left(e^{R} \frac{d}{d R} H^{(S)}\right)-\frac{\mathcal{K}^{2}}{4(D-3)^{2}} e^{-R} \frac{d}{d R} H^{(T r)}+\frac{\mathcal{K}}{2(D-3)} \nabla^{M} H_{M}^{(V)}$ \\
$+\mathcal{S}^{S_{2}}(R)+\frac{\mathcal{K}}{2(D-3)} e^{-R} \nabla \cdot u$ \\
$R^{S_{3}}=\left(\frac{\mathcal{K}^{2}}{2(D-3)^{2}}\right) e^{-2 R}\left(1-e^{R}\right) \frac{d}{d R}\left(e^{R} \frac{d H^{(S)}}{d R}\right)$ \\
$-\left(\frac{\mathcal{K}^{2}}{4(D-3)^{2}}\right) e^{-2 R}\left(1-e^{R}\right) \frac{\left.d H^{(T r}\right)}{d R}-\frac{\mathcal{K}}{2(D-3)} e^{-R} \nabla^{M} H_{M}^{(V)}+\mathcal{S}^{S_{3}}(R)+\frac{\mathcal{K}}{2(D-3)} e^{-2 R} \nabla \cdot u$ \\
$R^{S_{4}}=\left(\frac{\mathcal{K}^{2}}{(D-3)^{2}}\right) e^{-R} \frac{d}{d R}\left(e^{R} H^{(S)}\right)+\left(\frac{\mathcal{K}^{2}}{2(D-3)^{2}}\right) e^{-2 R}\left(1-e^{R}\right) \frac{d}{d R}\left(e^{R} \frac{d}{d R} H^{(T r)}\right)$ \\
$-\left(\frac{\mathcal{K}^{2}}{2(D-3)^{2}}\right) \frac{d H^{(T r)}}{d R}+\frac{\mathcal{K}}{D-3} \nabla^{M} H_{M}^{(V)}+\frac{2 \mathcal{K}}{D-3} \frac{d}{d R} \nabla^{M} H_{M}^{(V)}+\nabla^{M} \nabla^{N} H_{M N}^{(T)}+\mathcal{S}^{S_{4}}(R)-\frac{\mathcal{K}}{(D-3)} e^{-R} \nabla . u$ \\
\hline $\operatorname{Vector}$ sector \\
\hline$R_{M}^{V_{1}}=\left(\frac{\mathcal{K}^{2}}{2(D-3)^{2}}\right) e^{-R} \frac{d}{d R}\left(e^{R} \frac{d}{d R} H_{M}^{(V)}\right)+\frac{1}{2} \frac{\mathcal{K}}{(D-3)} \frac{d}{d R}\left(\nabla^{N} H_{N M}^{(T)}\right)+\mathcal{S}_{M}^{V_{1}}(R)$ \\
$R_{M}^{V_{2}}=\left(\frac{\mathcal{K}^{2}}{2(D-3)^{2}}\right) e^{-2 R}\left(1-e^{R}\right) \frac{d}{d R}\left(e^{R} \frac{d}{d R} H_{M}^{(V)}\right)+\mathcal{S}_{M}^{V_{2}}(R)$ \\
Tensor sector \\
\hline$R_{A B}^{T}=\left(\frac{-\mathcal{K}^{2}}{2(D-3)^{2}}\right) e^{-R} \frac{d}{d R}\left(\left(e^{R}-1\right) \frac{d H_{A B}^{(T)}}{d R}\right)+\mathcal{S}_{A B}^{T}(R)$ \\
\hline
\end{tabular}

Table 2. Expressions for basis of $R_{M N}$.

Let us imagine we have solved for our membrane metric at $(n-1)^{\text {th }}$ order in perturbation theory, and are now attempting to solve for the metric correction at $n^{\text {th }}$ order. If, in this process, we evaluate the constraint equation (2.17) and retain terms only up to $n^{\text {th }}$ order then we need use $G^{N L}$ on the r.h.s. of (2.17) only at zero order (i.e. from the metric (1.1)), because $E_{M N}$ is already of $n^{\text {th }}$ order. It follows that the $n^{\text {th }}$ order scalar and vector constraint equations are simply linear combinations of the $n^{\text {th }}$ order scalars and vectors listed in table 1. We will now determine the relevant linear combinations. In order to to this we first determine the $n^{\text {th }}$ order Ricci scalar $\tilde{R}$ as a linear combination of the scalars in table 1 .

$\tilde{R}=R_{A B} G^{A B}=\left(R^{A B} P_{A B}+\right.$ O.R.O $\left.\left(1-e^{-R}\right)+2 O \cdot R \cdot u\right)=\left(R^{S_{4}}+\left(1-e^{-R}\right) R^{S_{1}}+2 R^{S_{2}}\right)$

Using this equation we find

$$
\begin{aligned}
E_{M}^{(e c)} & =\left(R_{M N}-\frac{\tilde{R}}{2} G_{M N}\right) G^{N L} n_{L} \\
& =R_{M N} O^{N}\left(1-e^{-R}\right)+R_{M N} u^{N}-\frac{1}{2} \tilde{R} n_{M}
\end{aligned}
$$

By dotting (2.19) with $n$ and $u$ or by projecting it orthogonal to these vectors we finally obtain the $n^{\text {th }}$ order constraint equations written as linear combinations of the scalars and vectors in table 1.

$$
\begin{aligned}
& E^{S_{1}}=E_{M}^{(e c)} u^{M}=\left(1-e^{-R}\right) R^{S_{2}}+R^{S_{3}} \\
& E^{S_{2}}=E_{M}^{(e c)} O^{M}=\frac{1}{2}\left(\left(1-e^{-R}\right) R^{S_{1}}-R^{S_{4}}\right) \\
& E_{L}^{V}=E_{N}^{(e c)} \mathcal{P}_{L}^{N}=\left(1-e^{-R}\right) R_{L}^{V 1}+R_{L}^{V_{2}}
\end{aligned}
$$




\begin{tabular}{|c|}
\hline Vector constraint \\
\hline $\begin{aligned} & E_{M}^{V}=E_{N}^{(e c)} \mathcal{P}_{M}^{N}=\left(1-e^{-R}\right) R_{M}^{V_{1}}+R_{M}^{V_{2}} \\
= & \frac{1}{2} \frac{\mathcal{K}}{(D-3)}\left(1-e^{-R}\right) \frac{d}{d R}\left(\nabla^{A} H_{A M}^{(T)}\right)+\mathcal{V}_{M}^{V}(R)\end{aligned}$ \\
\hline Scalar constraint 1 \\
\hline $\begin{array}{c}E^{S_{1}}=E_{M}^{(e c)} u^{M}=\left(1-e^{-R}\right) R^{S_{2}}+R^{S_{3}} \\
=\frac{\mathcal{K}}{2(D-3)}\left(1-e^{R}\right) \frac{d}{d R}\left(\nabla^{M} H_{M}^{(V)}\right)^{-\frac{\mathcal{K}}{2(D-3)}} e^{-R} \nabla^{M} H_{M}^{(V)}+\mathcal{V}^{S 1}(R)+\frac{\mathcal{K}}{2(D-3)} e^{-R} \nabla \cdot u\end{array}$ \\
\hline Scalar constraint 2 \\
\hline $\begin{array}{c}E^{S_{2}}=E_{M}^{(e c)} O^{M}=\frac{1}{2}\left(\left(1-e^{-R}\right) R^{S_{1}}-R^{S_{4}}\right)=-\frac{\mathcal{K}}{2(D-3)} \frac{d}{d R}\left(\nabla^{M} H_{M}^{(V)}\right)-\frac{\mathcal{K}}{(D-3)} \nabla^{M} H_{M}^{(V)} \\
+\frac{\mathcal{K}^{2}}{4(D-3)^{2}}\left(2-e^{-R}\right) \frac{d}{d R} H^{(T r)}-\frac{\mathcal{K}^{2}}{2(D-3)^{2}}\left(\frac{d}{d R} H^{(S)}+H^{(S)}\right)-\frac{1}{2} \nabla_{M} \nabla_{N} H_{M N}^{(T)}+\mathcal{V}^{S 2}(R)+\frac{\mathcal{K}}{2(D-3)} e^{-R} \nabla \cdot u\end{array}$ \\
\hline
\end{tabular}

Table 3. Listing of constraint equations.

The explicit form of the $n^{\text {th }}$ order constraint equations is listed in table 3 above.

As in table 1, all fluctuation fields in table 3 should be taken to be of $n^{\text {th }}$ order. The source functions in table 3 are also of $n^{\text {th }}$ order and are given in terms of the sources in table 1 and the as yet unknown quantity $\nabla . u$ by

$$
\begin{aligned}
& \mathcal{V}^{S_{1}}(R)=\left(1-e^{-R}\right) \mathcal{S}^{S_{2}}(R)+\mathcal{S}^{S_{3}}(R) \\
& \mathcal{V}^{S_{2}}(R)=\frac{1}{2}\left[\left(1-e^{-R}\right) \mathcal{S}^{S_{1}}(R)-\mathcal{S}^{S_{4}}(R)\right] \\
& \mathcal{V}_{L}^{V}(R)=\left(1-e^{-R}\right) \mathcal{S}_{L}^{V_{1}}(R)+\mathcal{S}_{L}^{V_{2}}(R)
\end{aligned}
$$

Now it is well known that the Einstein tensor obeys the identity

$$
\nabla_{M} E^{M N}=0
$$

It is also well known (and easy to see) that this identity ensures that the 'normal' derivative of the constraint equations is a linear combination of the 'in plane' derivatives of Einstein's equations. ${ }^{10}$ Within the perturbation theory of interest to this paper the equation $(2.22)$ may be evaluated and projected onto its scalar and vector sectors and shown to be equivalent to the following relations

$$
\begin{aligned}
\frac{d}{d R} E_{M}^{V}+E_{M}^{V}+\frac{(D-3)}{\mathcal{K}} \nabla^{N} R_{N M}^{T} & =0 \\
\frac{d}{d R} E^{S_{1}}+E^{S_{1}}+\frac{(D-3)}{\mathcal{K}} \nabla^{N} R_{N}^{V_{2}} & =0 \\
\frac{d}{d R} E^{S_{2}}+E^{S_{2}}+\left(\frac{1}{2} R^{S_{1}}+R^{S_{2}}+\frac{1}{2} R^{S_{4}}\right)+\frac{(D-3)}{\mathcal{K}} \nabla^{N} R_{N}^{V_{1}} & =0
\end{aligned}
$$

\footnotetext{
${ }^{10}$ This is the fact that ensures that if all Einstein constraint equations are solved on one 'time' slice then they are automatically solved on the next 'time' slice. In other words, in order to solve Einstein's equations you need only solve the constraint equations on one time slice provided you solve the other equations lets call them the dynamical equations - everywhere.
} 
Using (2.20) the r.h.s. of these relations may be recast in the equivalent form

$$
\begin{aligned}
\frac{d}{d R} E_{M}^{V}+\left(1-e^{-R}\right) R_{M}^{V_{1}}+R_{M}^{V_{2}}+\frac{(D-3)}{\mathcal{K}} \nabla^{N} R_{N M}^{T} & =0 \\
\frac{d}{d R} E^{S_{1}}+\left(1-e^{-R}\right) R^{S_{2}}+R^{S_{3}}+\frac{(D-3)}{\mathcal{K}} \nabla^{N} R_{N}^{V_{2}} & =0 \\
\frac{d}{d R} E^{S_{2}}+\frac{1}{2} e^{-R} R^{S_{1}}+\left(1-e^{-R}\right) R^{S_{1}}+R^{S_{2}}+\frac{(D-3)}{\mathcal{K}} \nabla^{N} R_{N}^{V_{1}} & =0
\end{aligned}
$$

In either form these equations express the $R$ derivatives of the Einstein constraint equations (2.20) in terms of linear combinations of the Einstein equations. Using the explicit expressions in tables 2 and 3, it is possible to verify that the equations (2.23) are indeed obeyed, provided that the scalar and vector sources in table 2 and 3 are not all independent but are constrained by the following relations

$$
\begin{aligned}
& \frac{d}{d R} \mathcal{V}_{M}^{V}+\mathcal{V}_{M}^{V}+\frac{(D-3)}{\mathcal{K}} \nabla^{N} \mathcal{S}_{N M}^{T}=0 \\
& \frac{d}{d R} \mathcal{V}^{S_{1}}+\mathcal{V}^{S_{1}}+\frac{(D-3)}{\mathcal{K}} \nabla^{N} \mathcal{S}_{N}^{V_{2}}=0 \\
& \frac{d}{d R} \mathcal{V}^{S_{2}}+\mathcal{V}^{S_{2}}+\left[\frac{1}{2} \mathcal{S}^{S_{1}}+\left(\mathcal{S}^{S_{2}}+\frac{\mathcal{K}}{2(D-3)} e^{-R} \nabla \cdot u\right)+\frac{1}{2}\left(\mathcal{S}^{S_{4}}-\frac{\mathcal{K}}{(D-3)} e^{-R} \nabla . u\right)\right] \\
& +\frac{(D-3)}{\mathcal{K}} \nabla^{N} \mathcal{S}_{N}^{V_{1}}=0
\end{aligned}
$$

Note that we have two relations between the four scalar sources and one relation between the two vector sources in table 2. Note that the relations also involve the as yet unknown quantity $\nabla . u$. Later in this paper we will explicitly verify that the sources that appear in the first and second order calculation obey the relations (2.25). However we would like to emphasize here that these relations are necessarily obeyed at every order in perturbation theory.

\subsection{Choice of basis for the constraint and dynamical equations}

Because we have the linear relationship between constraint and dynamical equations we use the following basis for solving the scalar, vector and tensor fluctuations

$$
\begin{array}{ll}
\text { Tensor: } & R_{A B}^{T} \\
\text { Vector: } & R_{M}^{V_{2}}, E_{M}^{V} \\
\text { Scalar: } & R^{S_{1}}, R^{S_{2}}, \quad E^{S_{1}}, \quad E^{S_{2}}
\end{array}
$$

From now on we write every expression in this basis. The expressions that we get from Bianchi identities i.e. equations (2.23), (2.24) can be converted to the basis (2.26) as

$$
\begin{aligned}
\frac{d}{d R} E_{M}^{V}+E_{M}^{V}+\frac{(D-3)}{\mathcal{K}} \nabla^{N} R_{N M}^{T} & =0 \\
\frac{d}{d R} E^{S_{1}}+E^{S_{1}}+\frac{(D-3)}{\mathcal{K}} \nabla^{N} R_{N}^{V_{2}} & =0 \\
\frac{d}{d R} E^{S_{2}}+\left(1-\frac{1}{2} e^{-R}\right) R^{S_{1}}+R^{S_{2}}+\frac{1}{1-e^{-R}} \frac{(D-3)}{\mathcal{K}} \nabla^{M}\left(E_{M}^{V}-R_{M}^{V_{2}}\right) & =0
\end{aligned}
$$




\begin{tabular}{|c|}
\hline Scalar sector \\
\hline $\begin{array}{c}\mathcal{S}^{S_{1}}(R)=0 \\
\mathcal{S}^{S_{2}}(R)=\frac{\mathcal{K}}{2(D-3)} e^{-R} u \cdot K \cdot u-\frac{e^{-R}(-1+R)}{2} \frac{u . \nabla \mathcal{K}}{(D-3)}-\frac{\mathcal{K}^{2}}{2(D-3)^{2}} e^{-R}(-3+2 R) \\
\mathcal{S}^{S_{3}}(R)=\frac{1}{2 \mathcal{K}(D-3)} R e^{-R} \nabla^{2} \mathcal{K}-\frac{e^{-2 R}\left(-2+2 e^{R}+R\right)}{2} \frac{u . \nabla \mathcal{K}}{(D-3)}+\frac{\mathcal{K}^{2}}{2(D-3)^{2}} e^{-2 R}\left(3 e^{R}(R-1)-2 R+3\right) \\
\mathcal{S}^{S_{4}}(R)=e^{-R}(-1+R) \frac{u . \nabla \mathcal{K}}{(D-3)}+\frac{\mathcal{K}^{2}}{(D-3)^{2}} e^{-R}(-1+2 R)\end{array}$ \\
\hline Vector sector \\
\hline $\begin{array}{c}\mathcal{S}_{A}^{V_{1}}(R)=\frac{\mathcal{K}}{2(D-3)} e^{-R}\left(u^{M} K_{M N}-u^{M} \nabla_{M} u_{N}\right) \mathcal{P}_{A}^{N} \\
\mathcal{S}_{A}^{V_{2}}(R)=\frac{\mathcal{K}}{2(D-3)} e^{-2 R}\left(u^{M} K_{M N}-u^{M} \nabla_{M} u_{N}\right) \mathcal{P}_{A}^{N}+\frac{e^{-R}}{2}\left(\frac{\nabla^{2} u_{A}}{(D-3)}-\frac{\nabla_{A} \mathcal{K}}{(D-3)}\right)\end{array}$ \\
\hline Tensor sector \\
\hline $\mathcal{S}_{A B}^{T}(R)=0$ \\
\hline
\end{tabular}

Table 4. Sources of $R_{M N}$ equations at 1 st order.

The corresponding relationship between the sources is given by

$$
\begin{aligned}
\frac{d}{d R} \mathcal{V}_{M}^{V}+\mathcal{V}_{M}^{V}+\frac{(D-3)}{\mathcal{K}} \nabla^{N} \mathcal{S}_{N M}^{T} & =0 \\
\frac{d}{d R} \mathcal{V}^{S_{1}}+\mathcal{V}^{S_{1}}+\frac{(D-3)}{\mathcal{K}} \nabla^{N} \mathcal{S}_{N}^{V_{2}} & =0 \\
\frac{d}{d R} \mathcal{V}^{S_{2}}+\left(1-\frac{1}{2} e^{-R}\right) \mathcal{S}^{S_{1}}+\mathcal{S}^{S_{2}}+\frac{1}{1-e^{-R}} \frac{(D-3)}{\mathcal{K}} \nabla^{N}\left(\mathcal{V}_{N}^{V}-\mathcal{S}_{N}^{V_{2}}\right) & =0
\end{aligned}
$$

\section{Perturbation theory at first order}

In this section we will explicitly solve for the first order correction metric $h_{M N}^{(1)}$. However we will perform our analysis in a manner that makes the generalization to higher orders obvious.

\subsection{Listing first order source functions}

As we have explained in the previous section, the components of $R_{M N}^{1}$ are given in terms of $h_{M N}^{(1)}$ by the expressions in table 2 with particular values for the source functions in that table. By explicit calculation at first order we find that these source functions are given by the values listed in the table 4 .

Moreover the constraint equations take the form listed in table 3 with first order source functions listed in table 5. We list the corresponding sources to the constraint equations at 1 st order in table 5 . We have verified that our explicit expressions for the sources obey the constraints (2.25).

We now proceed to solve the metric corrections at 1st order i.e. $h_{M N}^{(1)}$. We impose the conditions (2.12) as discussed in section 2.4. 


\begin{tabular}{|c|}
\hline Vector constraint source \\
\hline $\mathcal{V}_{M}^{V}(R)=\frac{e^{-R}}{2}\left(\frac{\nabla^{2} u_{M}}{(D-3)}-\frac{\nabla_{M} \mathcal{K}}{(D-3)}+\frac{\mathcal{K}}{(D-3)}\left(u^{A} K_{A M}-u . \nabla u_{M}\right)\right)$ \\
\hline Scalar constraint 1 source \\
\hline $\mathcal{V}^{S_{1}}(R)=\frac{1}{2 \mathcal{K}(D-3)} R e^{-R} \nabla^{2} \mathcal{K}-\frac{-e^{-2 R}+e^{-R}(1+R)}{2} \frac{u . \nabla \mathcal{K}}{(D-3)}$ \\
$+\frac{\mathcal{K}}{2(D-3)} e^{-R}\left(1-e^{-R}\right) u . K . u+R e^{-R} \frac{\mathcal{K}^{2}}{2(D-3)^{2}}$ \\
\hline Scalar constraint 2 source \\
\hline $\mathcal{V}^{S_{2}}(R)=\frac{e^{-R}}{2}\left(\frac{\mathcal{K}^{2}}{(D-3)^{2}}(1-2 R)+\frac{u . \nabla \mathcal{K}}{(D-3)}(1-R)\right)$ \\
\hline
\end{tabular}

Table 5. Sources to constraint equations at 1st order.

\subsection{Tensor sector}

In this sector we have a single equation for the single variable $H_{M N}^{(T)}$. This equation is obtained by equating the last line of table 2 to zero and takes the form

$$
R_{A B}^{T}=e^{-R} \frac{d}{d R}\left(\left(e^{R}-1\right) \frac{d H_{A B}^{(T)}}{d R}\right)\left(\frac{-\mathcal{K}^{2}}{2(D-3)^{2}}\right)+\mathcal{S}_{A B}^{T}(R)=0
$$

where $\mathcal{S}_{A B}^{T}(R)$ is the source for the tensor sector. At first order it turns out that $\mathcal{S}_{A B}^{T}(R)=$ 0 (see table 5). In order to facilitate generalizations to higher orders however, in this subsection we will solve (3.1) for an arbitrary source function, and substitute $\mathcal{S}_{A B}^{T}(R)=0$ only at the end of the calculation.

Integrating (3.1) once we find

$$
\frac{d}{d R}\left(H_{A B}^{(T)}\right)=\left(\frac{-2(D-3)^{2}}{\mathcal{K}^{2}}\right) \frac{-1}{e^{R}-1} \int_{0}^{R} e^{x} \mathcal{S}_{A B}^{T}(x) d x
$$

The condition that $H_{A B}^{(T)}$ (and so r.h.s. of (3.2)) is regular at $R=0$ fixes the lower limit of the integral in (3.2). Integrating a second time we find

$$
\begin{aligned}
H_{A B}^{(T)} & =\left(\frac{-2(D-3)^{2}}{\mathcal{K}^{2}}\right) \int_{R}^{\infty} \frac{d y}{e^{y}-1} \int_{0}^{y} e^{x} \mathcal{S}_{A B}^{T}(x) d x \\
& =\left(\frac{2(D-3)^{2}}{\mathcal{K}^{2}}\right)\left[\log \left(1-e^{-R}\right) \int_{0}^{R} e^{x} S_{A B}^{T}(x) d x+\int_{R}^{\infty} \log \left(1-e^{-x}\right) e^{x} S_{A B}^{T}(x)\right]
\end{aligned}
$$

where the upper limit in the outer integral in (3.3) is fixed by the requirement that $H_{A B}^{(T)}$ decay at large $R$.

In summary, the tensor fluctuation $H_{A B}^{(T)}$ is given at any order, in terms of the tensor source function $\mathcal{S}_{A B}^{T}(x)$ at that order, by the expression (3.3). Note that $H_{A B}^{(T)}$ is uniquely determined by its source function; requirements of regularity at $R=0$ and decay at infinity unambiguously fix all integration constants in (3.1).

As we have mentioned above, at first order $\mathcal{S}_{A B}^{T, 1}(R)=0$. It follows from (3.3) that the first order tensor fluctuation $H_{A B}^{(T)}$ also vanishes and so

$$
H_{A B}^{(T, 1)}=0
$$




\subsection{Vector sector}

\subsubsection{Constraint equation and the membrane equation of motion}

In the vector sector we have two equations for the single variable $H_{M}^{(V)}$. The two equations may be chosen to be the vector constraint equation $E_{M}^{V}$ (see the first line of table 3 ) and the equation $R_{L}^{V_{2}}=0$ (see table 2 ).

One cannot, of course, solve two equations for a single variable unless one linear combination of the two equations is an identity. Indeed the first equation of (2.27)

$$
\frac{d}{d R} E_{M}^{V}+E_{M}^{V}+\frac{(D-3)}{\mathcal{K}} \nabla^{N} R_{N M}^{T}=0
$$

asserts that the vector constraint equation is automatically solved at all values of $R$ if its solved at one value of $R$ (we use here that we have already solved the tensor equation so that $\left.R_{A B}^{T}=0\right)$.

We will find it convenient to solve the vector constraint equation at $R=0$. From table 3 we see that

$$
E_{M}^{V}=\frac{1}{2} \frac{\mathcal{K}}{(D-3)}\left(1-e^{-R}\right) \frac{d}{d R}\left(\frac{\nabla^{M} H_{M N}^{(T)}}{(D-3)}\right)+\mathcal{V}_{M}^{V}(R)
$$

At $R=0$

$$
E_{M}^{V}=\mathcal{V}_{M}^{V}(0)
$$

It follows that the constraint equation is solved at $R=0$ if and only if $\mathcal{V}_{M}^{V}(0)$ vanishes (here we use the fact that $H_{M N}^{(T)}$ is regular at $R=0$; see the previous subsection). This requirement is a statement of the membrane equations of motion.

We would like to reemphasize that the membrane equations of motion at $n^{\text {th }}$ order are obtained simply by evaluating the $n^{\text {th }}$ order vector constraint equation at $R=0$. At $R=0$ this equation is independent of all the unknown $n^{\text {th }}$ order fluctuation fields. As a consequence the membrane equations of motion may be obtained at $n^{\text {th }}$ order before solving for the fluctuation fields at $n^{\text {th }}$ order, as in studies of the fluid gravity correspondence.

The analysis presented in this subsection so far has been valid at every order in perturbation theory. Specializing now to the first order, we read off the value of $\mathcal{V}_{M}^{V}(0)$ from table 5. Equating this expression to zero we find the first order membrane equation of motion

$$
\left(\frac{\nabla^{2} u_{A}}{\mathcal{K}}-\frac{\nabla_{A} \mathcal{K}}{\mathcal{K}}+u_{C} K_{A}^{C}-u . \nabla u_{A}\right) \mathcal{P}_{B}^{A}=0
$$

While all fields in (3.6) live in the full bulk spacetime $R^{D-1,1}$, and all derivatives in that equation are bulk spacetime derivatives, the equation (3.6) itself holds only on the membrane surface $\psi=1$. Using the subsidiary conditions (2.4) it is possible to rewrite (3.6) as an equation restricted to the membrane. As demonstrated in [9] the equation of motion of motion turns out to take exactly the same form as (3.6) in this language. In other words (3.6) also holds true if we think of $K_{M N}$ and $u_{M}$ as membrane world volume fields, and regard every derivative in that equation as a covariant derivative on the membrane world volume. 


\subsubsection{Solving for the vector fluctuation}

As we have explained in the previous subsubsection, the constraint vector equation is automatically solved at every $R$ provided the membrane equation is obeyed. Assuming this is the case, we have already solved one of the two vector equations.

In order to solve for the unknown function, $H_{M}^{(V)}$, in the vector sector, we now turn to the second vector equation $R_{L}^{V_{2}}=0$. This equation takes the form

$$
\left(\frac{-\mathcal{K}^{2}}{2(D-3)^{2}}\right) e^{-2 R}\left(-1+e^{R}\right) \frac{d}{d R}\left(e^{R} \frac{d}{d R} H_{M}^{(V)}\right)+\mathcal{S}_{M}^{V_{2}}(R)=0
$$

As in the previous subsection we will proceed to solve (3.7) for an arbitrary source function, plugging in the first order result for the source

$$
\mathcal{S}_{A}^{V_{2}, 1}(R)=-\frac{\mathcal{K}}{2(D-3)} e^{-2 R}\left(-1+e^{R}\right)\left(u^{M} K_{M N}-u^{M} \nabla_{M} u_{N}\right) \mathcal{P}_{A}^{N}
$$

only at the end of the computation.

Notice that the 1.h.s. of (3.7) vanishes at $R=0$. It follows that (3.7) admits regular solutions if and only if $\mathcal{S}_{M}^{V_{2}}(R)$ also vanishes at $R=0$. It would naively seem that this requirement imposes a new constraint on membrane data, independent of (3.6). ${ }^{11}$ However it turns out that the vanishing of $\mathcal{S}_{M}^{V_{2}}(R)$ is automatic; indeed it follows from (2.20) that $R_{M}^{V_{2}}$ is simply identical to the vector constraint equation $E_{M}^{V}$ at $R=0$. It follows as a consequence that $\mathcal{S}_{M}^{V_{2}}(R)$ is proportional to the l.h.s. of (3.6) at $R=0 .{ }^{12}$

Using the fact that $\mathcal{S}_{M}^{V_{2}, 1}(0)$ vanishes, we integrate (3.7) once to find

$$
e^{R} \frac{d}{d R} H_{M}^{(V)}=\left(\frac{-2(D-3)^{2}}{\mathcal{K}^{2}}\right)\left[\int_{0}^{R}\left(\frac{-e^{y}}{1-e^{-y}}\right) \mathcal{S}_{M}^{V_{2}}(y) d y+C_{M}^{V_{2}}\right]
$$

where $C_{M}^{V_{2}}$ is an as yet undetermined integration constant. Integrating a second time we find

$$
H_{M}^{(V)}=\left(\frac{2(D-3)^{2}}{\mathcal{K}^{2}}\right) \int_{R}^{\infty} e^{-x}\left[\int_{0}^{x}\left(\frac{-e^{y}}{1-e^{-y}}\right) \mathcal{S}_{M}^{V_{2}}(y) d y\right] d x-C_{M}^{V_{2}} e^{-R}
$$

The upper limit on the the outer integral of (3.10) has been determined from the requirement that $H_{M}^{(V)}$ vanishes at large $R$. The expression for $H_{M}^{V}$ may be simplified by integrating by parts; we find

$$
H_{M}^{(V)}(R)=\left(\frac{2(D-3)^{2}}{\mathcal{K}^{2}}\right)\left(e^{-R} \int_{0}^{R}\left(\frac{-e^{x}}{1-e^{-x}}\right) \mathcal{S}_{M}^{V_{2}}(x) d x-\int_{R}^{\infty} \frac{\mathcal{S}_{M}^{V_{2}}(x)}{1-e^{-x}}\right)-C_{M}^{V_{2}} e^{-R}
$$

In particular that

$$
H_{M}^{(V)}(0)=-\left(\frac{2(D-3)^{2}}{\mathcal{K}^{2}}\right) \int_{0}^{\infty} \frac{\mathcal{S}_{M}^{V_{2}}(x)}{1-e^{-x}}-C_{M}^{V_{2}}
$$

\footnotetext{
${ }^{11}$ Had this step of the programme imposed a new constraint, we would have obtained a new membrane equation - and so obtained more membrane equations than membrane variables, leading to an inconsistent dynamical system.

${ }^{12}$ To see this we note that (3.7) reduces to $\mathcal{S}_{M}^{V_{2}}(R)$ at $R=0$ while $E_{M}^{V}$ reduces to the l.h.s. of (3.6) at $R=0$.
} 
It follows (see (2.12)) that

$$
C_{M}^{V_{2}}=-\left(\frac{2(D-3)^{2}}{\mathcal{K}^{2}}\right) \int_{0}^{\infty} \frac{\mathcal{S}_{M}^{V_{2}}(x)}{1-e^{-x}}
$$

so that

$$
H_{M}^{(V)}(R)=\left(\frac{2(D-3)^{2}}{\mathcal{K}^{2}}\right)\left(e^{-R} \int_{0}^{R}\left(\frac{-e^{x}}{1-e^{-x}}\right) \mathcal{S}_{M}^{V_{2}}(x) d x-\int_{R}^{\infty} \frac{\mathcal{S}_{M}^{V_{2}}(x)}{1-e^{-x}}+e^{-R} \int_{0}^{\infty} \frac{\mathcal{S}_{M}^{V_{2}}(x)}{1-e^{-x}}\right)
$$

The expression (3.14) is our final expression for $H_{M}^{(V)}(R)$ at any order in perturbation theory in terms of the source function at that order. Note that $H_{M}^{(V)}(R)$ is uniquely determined in terms of its source function; the integration constants in (3.7) are uniquely determined by the requirement that $H_{M}^{(V)}(R)$ vanish at infinity and that (2.12) is obeyed at $R=0$.

Plugging the first order expression for the source (3.8) into (3.14), at first order we find

$$
H_{M}^{(V, 1)}=\frac{(D-3)}{\mathcal{K}} R e^{-R}\left(u^{A} K_{A N}-u^{A} \nabla_{A} u_{N}\right) P_{M}^{N}
$$

\subsection{Scalar sector}

In the scalar sector we have four equations for the two variables $H^{(T r)}$ and $H^{(S)}$. As a basis for the four equations we find it convenient to use the two scalar constraint equations $E^{S_{1}}$ and $E^{S_{2}}$ (see table 3 ) together with the two additional equations $R^{S_{1}}=0$ and $R^{S_{2}}=0$ (see table 1).

\subsubsection{Constraint equations and $\nabla . u$}

As in the previous subsection it is consistent to have four equations for two variables only if two of the four equations are identities. The last two equations in (2.27)

$$
\begin{aligned}
\frac{d}{d R} E^{S_{1}}+E^{S_{1}}+\frac{(D-3)}{\mathcal{K}} \nabla^{N} R_{N}^{V_{2}} & =0 \\
\frac{d}{d R} E^{S_{2}}+\left(1-\frac{1}{2} e^{-R}\right) R^{S_{1}}+R^{S_{2}}+\frac{(D-3)}{\mathcal{K}} \frac{1}{1-e^{-R}} \nabla^{M}\left(E_{M}^{V}-R_{M}^{V_{2}}\right) & =0
\end{aligned}
$$

assert that this is indeed the case. As we have already solved the vector sector at $n^{\text {th }}$ order $R_{N}^{V_{2}}$ vanishes. It follows that the first equation in (3.16) asserts that if $E^{S_{1}}$ is solved at any $R$ it is automatically solved at every $R$. When evaluated at $R=0$ this equation reduces to the condition

$$
\mathcal{V}^{S_{1}}(0)+\frac{\mathcal{K}}{2(D-3)} \nabla \cdot u=0
$$

Recall that at leading order $\nabla . u=0 .(3.17)$ determines the correction to this statement at subleading orders.

As in the previous subsection we emphasize that the expression for $\nabla . u$ at $n^{\text {th }}$ order is determined simply by evaluating the $n^{\text {th }}$ order constraint equation $E^{S_{1}}$ at $R=0$. In order to obtain this correction we do not need to solve for any of the $n^{\text {th }}$ order fluctuation fields, all of which drop out in $E^{S_{1}}$ evaluated at $R=0$. 
The analysis of this subsection has, so far, been valid at every order in perturbation theory. Specializing to first order it is easily verified from table 5 that $\mathcal{V}^{S_{1}}(0)=0$. It follows that the zero order relation $\nabla \cdot u=0$ is uncorrected at first order (since $(\nabla \cdot u)_{0}=$ $\left.\mathcal{V}^{S_{1}}(0)=0\right)$. As we will see in the next section, the situation is different at second order.

The constraint equation $E^{S_{2}}$ plays a distinct logical role from $E^{S_{1}}$ in our perturbative programme. Once the tensor and vector equations had been solved, (3.16) assured us that $E^{S_{1}}(R)$ obeys a homogeneous differential equation in $R$ (see $(2.23)$ which makes no reference to any of the other equations in the scalar sector. On the other hand the differential equation obeyed by $E^{S_{2}}$ involves the other scalar equations (see the last equation in (2.24)). The most useful way to view the last equation in (2.24) is as follows. It might, a priori, have seemed that we have 4 equations in the scalar sector. We have already dealt with $E^{S_{1}}$ above leaving behind a three dimensional space of equations. A useful basis for this space is given by $E^{S_{2}}, R^{S_{1}}$ and $R^{S_{2}}$. The last equation in (2.24) allows us to eliminate $R^{S_{2}}$ from this basis. In order to complete solving in the scalar sector we need only solve the equations $E^{S_{2}}$, $R^{S_{1}}$. In other words the constraint equation $E^{S_{2}}$ does not constrain data: instead it may be used to solve for the scalar fluctuation. We turn to this task in the next subsubsection.

\subsubsection{Solving for the scalar fluctuations}

The equation $R^{S_{1}}$

$$
R^{S_{1}}=\left(\frac{-\mathcal{K}^{2}}{2(D-3)^{2}}\right) \frac{d^{2} H^{(T r)}}{d R^{2}}+\mathcal{S}^{S_{1}}(R)=0
$$

is easily solved. Integrating the above equation once we get

$$
\frac{d H^{(T r)}}{d R}=\left(\frac{-2(D-3)^{2}}{\mathcal{K}^{2}}\right) \int_{R}^{\infty} d x \mathcal{S}^{S_{1}}(x)
$$

Where we have fixed the boundary condition from the requirement that $H^{(T r)}$ and so its derivative $\frac{d H^{(T r)}}{d R}=0$ vanish at large $R$. Integrating this equation once again we have

$$
\begin{aligned}
H^{(T r)} & =\left(\frac{2(D-3)^{2}}{\mathcal{K}^{2}}\right) \int_{R}^{\infty} d y \int_{y}^{\infty} d x \mathcal{S}^{S_{1}}(x) \\
& =\left(\frac{2(D-3)^{2}}{\mathcal{K}^{2}}\right)\left[-R \int_{R}^{\infty} d x \mathcal{S}^{S_{1}}(x)+\int_{R}^{\infty} d x x \mathcal{S}^{S_{1}}(x)\right]
\end{aligned}
$$

where, once again we have fixed the integration constant from the requirement that $H^{(T r)}=$ 0 at large $R$.

Specializing now to first order we note $\mathcal{S}^{S_{1}, 1}=0$ so that

$$
H^{(T r, 1)}=0
$$

The equation $E^{S_{2}}$ takes the form

$$
\begin{aligned}
\frac{d}{d R}\left(H^{(S)} e^{R}\right)= & \frac{2(D-3)^{2}}{\mathcal{K}^{2}} e^{R} \mathcal{S}_{S}(R) \quad \text { where } \\
\mathcal{S}_{S}(R)= & -\frac{\mathcal{K}}{2(D-3)} \frac{d}{d R}\left(\nabla^{M} H_{M}^{(V)}\right)-\frac{\mathcal{K}}{(D-3)} \nabla^{M} H_{M}^{(V)} \\
& +\frac{\mathcal{K}^{2}}{4(D-3)^{2}}\left(2-e^{-R}\right) \frac{d}{d R} H^{(T r)}-\frac{1}{2} \nabla^{M} \nabla^{N} H_{M N}^{(T)}+\mathcal{V}^{S_{2}}(R)+\frac{\mathcal{K}}{2(D-3)} e^{-R} \nabla . u
\end{aligned}
$$


Plugging in the already obtained expressions of $H_{M}^{(V)}, H_{M N}^{(T)}, H^{(T r)}$ (see (3.14), (3.20) and (3.3)) and using (2.28), the source function $\mathcal{S}_{S}(R)$ can be rewritten as a linear functional of the elementary sources $\mathcal{S}^{S_{1}}, \mathcal{S}^{S_{2}}$ and $\mathcal{V}^{S_{1}}{ }^{13}$ Upon simplifying (by integrating by parts on several occasions) we find

$$
\begin{aligned}
\mathcal{S}_{S}(R)= & \int_{R}^{\infty} \mathcal{S}^{S_{2}}(x) d x+\frac{1}{2} \int_{R}^{\infty}\left(2-e^{-x}\right) \mathcal{S}^{S_{1}}(x) d x-\frac{1}{2}\left(2-e^{-R}\right) \int_{R}^{\infty} \mathcal{S}^{S_{1}}(x) d x \\
& -\left(1-e^{-R}\right) \int_{R}^{\infty}\left(\frac{e^{x}\left(\mathcal{V}^{S_{1}}{ }^{\prime}(x)+\mathcal{V}^{S_{1}}(x)\right)}{\left(e^{x}-1\right)} d x\right) d y-\mathcal{V}^{S_{1}}(R)+e^{-R} \mathcal{V}^{S_{1}}(0) \\
& +\log \left(1-e^{-R}\right)\left(\mathcal{V}^{S_{1}{ }^{\prime}}(0)+\mathcal{V}^{S_{1}}(0)\right)+(\nabla \cdot u) \frac{\mathcal{K} e^{-R}}{2(D-3)}
\end{aligned}
$$

We note that $\mathcal{S}_{S}$ is analytic at $R=0$ if and only if

$$
\mathcal{V}^{S_{1}}(0)+\mathcal{V}^{S_{1}}(0)=0
$$

This condition is, in fact, automatic. It follows from the second of (2.28) that the l.h.s. of (3.24) is proportional to $\nabla^{N} \mathcal{S}_{N}^{V_{2}}(0)$. We have already argued, however, that $\mathcal{S}_{N}^{V_{2}}$ vanishes at $R=0$. Since this condition holds at every point on the membrane, it follows also that $\nabla^{N} \mathcal{S}_{N}^{V_{2}}(0)=0$ establishing $(3.24){ }^{14}$ find

Plugging (3.23) into (3.22), integrating (and simplifying using integration by parts) we

$$
\begin{aligned}
H_{S}(R)= & \frac{2(D-3)^{2}}{\mathcal{K}^{2}} e^{-R}\left(\frac{(\mathcal{K}(\nabla \cdot u)) R}{2(D-3)}+e^{R} \int_{R}^{\infty} \mathcal{S}^{S_{2}}(x) d x-\int_{0}^{\infty} \mathcal{S}^{S_{2}}(x) d x+\int_{0}^{R} e^{x} \mathcal{S}^{S_{2}}(x) d x\right. \\
& +\frac{e^{R}}{2} \int_{R}^{\infty}\left(2-e^{-x}\right) \mathcal{S}^{S_{1}}(x) d x+\frac{1}{2} \int_{0}^{R} e^{x}\left(2-e^{-x}\right) \mathcal{S}^{S_{1}}(x) d x-\frac{1}{2} \int_{0}^{\infty}\left(2-e^{-x}\right) \mathcal{S}^{S_{1}}(x) d x \\
& -\frac{1}{2}\left(2 e^{R}-R\right) \int_{R}^{\infty} \mathcal{S}^{S_{1}}(x) d x+\int_{0}^{\infty} \mathcal{S}^{S_{1}}(x) d x-\frac{1}{2} \int_{0}^{R}\left(2 e^{y}-y\right) \mathcal{S}^{S_{1}}(x) d x \\
& \left.-\int_{0}^{R}\left(e^{y}-1\right) \int_{y}^{\infty}\left(\frac{e^{x}\left(\mathcal{V}^{S_{1}}{ }^{\prime}(x)+\mathcal{V}^{S_{1}}(x)\right)}{\left(e^{x}-1\right)} d x\right) d y-\int_{0}^{R} e^{x} \mathcal{V}^{S_{1}}(x) d x+R \mathcal{V}^{S_{1}}(0)\right)
\end{aligned}
$$

Explicitly at first order

$$
H^{(S, 1)}=\frac{D-3}{\mathcal{K}} R e^{-R}\left(R\left(-\frac{\mathcal{K}}{D-3}-\frac{u \cdot \nabla \mathcal{K}}{\mathcal{K}}+\frac{u \cdot K \cdot u}{2}\right)+\left(\frac{\mathcal{K}}{D-3}+u \cdot K \cdot u\right)\right)
$$

\footnotetext{
${ }^{13}$ It turns out that all dependence on the fourth independent scalar source, $\mathcal{V}^{S_{2}}$ cancels.

${ }^{14} \mathrm{In}$ studies of the fluid gravity correspondence a derivative of the equation of the $n^{\text {th }}$ order equation contributes to sources only at $(n+1)^{\text {th }}$ order in the derivative expansion. In the large $D$ expansion of this paper, however, the suppression in order resulting from using an extra derivative can be compensated for by an enhancement in order resulting from the contraction of a spacetime index. Consequently the equation of motion and its contracted derivatives are of the same order in the large $D$ expansion.
} 


\subsection{Final result for the first order metric}

After integrating the ordinary differential equations corresponding to Einstein's equations and imposing the condition that the metric is regular at the horizon, matches flat space at the end of the membrane region and (2.12), we get the following solutions for the various components of the metric correction.

$$
\begin{aligned}
H_{M N}^{(T, 1)} & =0 \\
H^{(T r, 1)} & =0 \\
H_{M}^{(V, 1)} & =\frac{(D-3)}{\mathcal{K}} R e^{-R}\left(u^{A} K_{A L}-u^{A} \nabla_{A} u_{L}\right) \mathcal{P}_{M}^{L} \\
H^{(S, 1)} & =\frac{D-3}{\mathcal{K}} R e^{-R}\left(R\left(-\frac{\mathcal{K}}{D-3}-\frac{u \cdot \nabla \mathcal{K}}{\mathcal{K}}+\frac{u \cdot K \cdot u}{2}\right)+\left(\frac{\mathcal{K}}{D-3}+u \cdot K \cdot u\right)\right)
\end{aligned}
$$

Thus we can write the 1 st order corrected metric as

$$
\begin{aligned}
g_{M N} & =\eta_{M N}+\frac{O_{M} O_{N}}{\psi^{D-3}} \\
& +\frac{1}{D-3}\left[\frac{D-3}{\mathcal{K}} R e^{-R}\left(R\left(-\frac{\mathcal{K}}{D-3}-\frac{u \cdot \nabla \mathcal{K}}{\mathcal{K}}+\frac{u \cdot K \cdot u}{2}\right)+\left(\frac{\mathcal{K}}{D-3}+u \cdot K \cdot u\right)\right) O_{M} O_{N}\right. \\
& \left.+\frac{(D-3)}{\mathcal{K}} R^{-R}\left(u^{A} K_{A L}-u^{A} \nabla_{A} u_{L}\right) P_{(M}^{L} O_{N)}\right]
\end{aligned}
$$

\section{2nd order solution}

The metric (3.28) solves Einstein equation to first subleading order. In this section we implement the perturbative procedure to one higher order. In other words we determine the correction $H_{M N}^{(2)}$ in a way that ensures that $R_{A B}$ evaluated on the corrected metric is of order $1 / D$ (more precisely that $R_{A B} R^{A B}$ is of order $1 / D^{2}$ ).

The procedure we follow is exactly that of the previous section: in fact second order corrections to the metric are given directly by the formulae of the previous subsection with one modification: we need to use the second order rather than first order source functions. In other words the computation at second order boils down entirely to determining the second order sources.

In order to determine the sources at second order we plug the first order corrected metric (3.28) together with an as yet undetermined second order correction $h_{M N}^{2}$ into Einstein's equations. We use the fact that the shape and velocity functions in the first order corrected metric obey the equation of motion

$$
\left(\frac{\nabla^{2} u_{A}}{\mathcal{K}}-\frac{\nabla_{A} \mathcal{K}}{\mathcal{K}}+u_{C} K_{A}^{C}-u . \nabla u_{A}\right) \mathcal{P}_{B}^{A}+\frac{1}{D} \mathcal{E}_{A} \mathcal{P}_{B}^{A}=0
$$

where $\mathcal{E}_{B}$ is an as yet undetermined '2nd order' correction to the equations of motion. As in the previous subsection we solve the equations in the neighbourhood of a particular point on the event horizon. In our analysis, however, we use the fact that the membrane equations of motion (4.1) are obeyed not just at the particular point we are expanding 
about but everywhere on the membrane. In other words we use the fact that the derivative of (4.1) vanishes at the point of interest. Finally we also use the fact that $\nabla . u$ is an as yet undetermined quantity of order $1 / D$.

We find by explicit computation that the curvature components listed table 1 do indeed take the form listed in table 2, 3 once all metric fluctuation fields in that table are identified with second order fluctuations. Our explicit computations also yield explicit expressions for all the second order source functions. We present an explicit listing of these source functions in tables 6 and 7 in the appendix.

In the rest of this section we obtain the second order correction to the metric by inserting the second order sources into general integral formulae of the previous section and performing all integrals.

\subsection{Constraints on membrane data}

\subsubsection{Correction to the membrane equations from the vector sector}

As in the previous subsection (3.5) guarantees that the vector constraint equation $E_{M}^{V}=0$ is solved at any $R$ if the equation is obeyed at $R=0$. As in the previous subsection the constraint equation at $R=0$ is independent of the second order fluctuation fields. From table 7 we see that this constraint equation at $R=0$ determines $-\frac{1}{D} \mathcal{E}_{A} \mathcal{P}_{B}^{A}-$ the second order correction to the membrane equation of motion - in terms of appropriate expressions involving the membrane extrinsic curvature and velocity fields. Adding these correction terms to the first order membrane equation (1.3) we recover the second order corrected membrane equation

$$
\begin{aligned}
& {\left[\frac{\nabla^{2} u}{\mathcal{K}}-\frac{\nabla \mathcal{K}}{\mathcal{K}}+u \cdot K-(u \cdot \nabla) u\right] \cdot \mathcal{P}+\left[\frac{\nabla^{2} \nabla^{2} u}{\mathcal{K}^{3}}-\frac{\nabla\left(\nabla^{2} \mathcal{K}\right)}{\mathcal{K}^{3}}\right.} \\
& \quad+3 \frac{(u \cdot K \cdot u)(u \cdot \nabla u)}{\mathcal{K}}-3 \frac{(u \cdot K \cdot u)(u \cdot \nabla n)}{\mathcal{K}}-6 \frac{\left(u \cdot\left(\nabla^{2} n\right)\right)(u \cdot \nabla u)}{\mathcal{K}^{2}} \\
& \left.\quad+6 \frac{\left(u \cdot\left(\nabla^{2} n\right)\right)(u \cdot \nabla n)}{\mathcal{K}^{2}}+\frac{3}{D-3} u \cdot \nabla u-\frac{3}{D-3} u \cdot \nabla n\right] \cdot \mathcal{P}=0
\end{aligned}
$$

where

$$
\mathcal{P}^{A B}=\eta^{A B}-n^{A} n^{B}+u^{A} u^{B}
$$

The 1st square bracket in (4.2) is simply the 1st order equation of motion while the 2 nd square bracket represents subleading corrections. ${ }^{15}$

We would like, however, to emphasize an important technical point. All the fields in (4.2) are assumed to live in all of the embedding flat spacetime; they are extended off

\footnotetext{
${ }^{15}$ Note that we can write the equation (4.2) in a nicer looking form by using the subsidiary conditions (2.4), divergence of first order membrane equation of motion (1.3) and divergence of velocity condition (1.5). The form is

$$
\left(\frac{\nabla^{2} O}{\nabla \cdot O}+O . \nabla O\right) \cdot \mathcal{P}+\left(\frac{\nabla^{2} \nabla^{2} O}{(\nabla \cdot O)^{3}}+3 \frac{\nabla^{2}(\nabla . O)}{(\nabla \cdot O)^{3}} O \cdot \nabla O\right) \cdot \mathcal{P}=0 .
$$


the surface of the membrane by the subsidiary conditions listed earlier in this paper. While all covariant derivatives listed in (4.2) are evaluated on the surface of the membrane, they act on fields defined in all of spacetime.

As the membrane equations of motion are intrinsic to the membrane, it is clearly unnatural to write them in terms of spacetime derivatives of an essentially arbitrary extension of membrane fields into the embedding spacetime. The equation of motion (4.2) can be rewritten so that all fields in that equation are purely membrane world volume fields, and every derivative in the equation is a covariant derivative on the membrane world volume. We now explain how this is done.

The relationship between the bulk covariant derivatives of tensors (e.g. $u_{M}$ ) and membrane worldvolume derivatives of the same quantities is quite straightforward when no more than one derivative acts on the same object. The spacetime covariant derivative is obtained from the corresponding bulk quantity by projecting every index (not just the derivative indices) onto the membrane world volume. However this relationship is more complicated when we have two or more derivatives acting on the same object; the reason for the additional complication is that the formula for multiple worldvolume covariant derivatives involves inserting projectors at each step (when you define the first derivative in terms of bulk derivatives, then again when you define the second derivative in terms of bulk derivatives etc); when such expressions are opened out, outer derivatives act on projectors used to define the inner derivatives. Tracing through the required algebra we find that the corrected second order membrane equation of motion, written in terms of fields and covariant derivatives that live purely on the membrane world volume, takes the form

$$
\begin{aligned}
& {\left[\frac{\nabla^{2} u_{A}}{\mathcal{K}}-\frac{\nabla_{A} \mathcal{K}}{\mathcal{K}}+u^{B} K_{B A}-u \cdot \nabla u_{A}\right] \mathcal{P}_{C}^{A}} \\
& +\left[\left(-\frac{u^{C} K_{C B} K_{A}^{B}}{\mathcal{K}}\right)+\left(\frac{\nabla^{2} \nabla^{2} u_{A}}{\mathcal{K}^{3}}-\frac{u \cdot \nabla \mathcal{K} \nabla_{A} \mathcal{K}}{\mathcal{K}^{3}}-\frac{\nabla^{B} \mathcal{K} \nabla_{B} u_{A}}{\mathcal{K}^{2}}-2 \frac{K^{C D} \nabla_{C} \nabla_{D} u_{A}}{\mathcal{K}^{2}}\right)\right. \\
& +\left(-\frac{\nabla_{A} \nabla^{2} \mathcal{K}}{\mathcal{K}^{3}}+\frac{\nabla_{A}\left(K_{B C} K^{B C} \mathcal{K}\right)}{\mathcal{K}^{3}}\right)+3 \frac{(u \cdot K \cdot u)\left(u \cdot \nabla u_{A}\right)}{\mathcal{K}}-3 \frac{(u \cdot K \cdot u)\left(u^{B} K_{B A}\right)}{\mathcal{K}} \\
& \left.-6 \frac{(u \cdot \nabla \mathcal{K})\left(u \cdot \nabla u_{A}\right)}{\mathcal{K}^{2}}+6 \frac{(u \cdot \nabla \mathcal{K})\left(u^{B} K_{B A}\right)}{\mathcal{K}^{2}}+\frac{3}{D-3} u \cdot \nabla u_{A}-\frac{3}{D-3} u^{B} K_{B A}\right] \mathcal{P}_{C}^{A}=0
\end{aligned}
$$

The projector $\mathcal{P}^{A B}$ used in this equation

$$
\mathcal{P}^{A B}=\tilde{g}^{A B}+u^{A} u^{B}
$$

where $\tilde{g}^{A B}$ is the induced metric on the world volume of the membrane.

The equation (4.5) can be slightly simplified as follows. Let us first note that (4.5) takes the schematic form

$$
F^{A}+\frac{S^{A}}{\mathcal{K}}=0
$$

where $F^{A}$ is the first order contribution to the equation of motion (the first line of (4.5)) while $\frac{S^{A}}{\mathcal{K}}$ is the second order contribution (the second-fourth lines of (4.5)). $F^{A}$ and $S^{A}$ are each vector fields of order unity. 
Let us now consider the modified equation of motion

$$
F^{A}+\frac{S^{A}}{\mathcal{K}}+\nabla \cdot F \frac{\zeta^{A}}{\mathcal{K}^{2}}=0
$$

where $\zeta^{A}$ is any vector field of order unity. As $\nabla . F$ is naively of order $D$, the difference between the equations (4.8) and (4.7) is naively of order $\frac{1}{D}$ suggesting that (4.7) and (4.8) differ at first subleading order. This is not the case. By taking a divergence of either (4.7) or (4.8), the reader can easily convince herself that, onshell, $\nabla . F$ is of order unity (rather than the naive estimate of order $D$ ). If follows that (4.8) and (4.7) actually differ only at second subleading order $\left(\frac{1}{D^{2}}\right)$ and are equivalent at first subleading order. We are thus allowed to simplify (4.5) by adding any expression of the form $\nabla \cdot F \frac{\zeta^{A}}{\mathcal{K}^{2}}$ to it.

Now it was demonstrated in [9] that

$$
\frac{\nabla . F}{\mathcal{K}}=\frac{\nabla^{2} \mathcal{K}}{\mathcal{K}^{2}}-2 \frac{u . \nabla \mathcal{K}}{\mathcal{K}}+u . K . u
$$

Using this relation and making the the choice

$$
\zeta^{A}=-3\left((u \cdot \nabla u)_{A}-u_{B} K_{A}^{B}\right)
$$

we find that (4.5) is equivalent to (4.8) whose explicit form is

$$
\begin{aligned}
& {\left[\frac{\nabla^{2} u_{A}}{\mathcal{K}}-\frac{\nabla_{A} \mathcal{K}}{\mathcal{K}}+u^{B} K_{B A}-u \cdot \nabla u_{A}\right] \mathcal{P}_{C}^{A}} \\
& +\left[\left(-\frac{u^{C} K_{C B} K_{A}^{B}}{\mathcal{K}}\right)+\left(\frac{\nabla^{2} \nabla^{2} u_{A}}{\mathcal{K}^{3}}-\frac{u \cdot \nabla \mathcal{K} \nabla_{A} \mathcal{K}}{\mathcal{K}^{3}}-\frac{\nabla^{B} \mathcal{K} \nabla_{B} u_{A}}{\mathcal{K}^{2}}-2 \frac{K^{C D} \nabla_{C} \nabla_{D} u_{A}}{\mathcal{K}^{2}}\right)\right. \\
& +\left(-\frac{\nabla_{A} \nabla^{2} \mathcal{K}}{\mathcal{K}^{3}}+\frac{\nabla_{A}\left(K_{B C} K^{B C} \mathcal{K}\right)}{\mathcal{K}^{3}}\right)-3 \frac{\nabla^{2} \mathcal{K} u \cdot \nabla u_{A}}{\mathcal{K}^{3}}+3 \frac{\nabla^{2} \mathcal{K} u^{B} K_{B A}}{\mathcal{K}^{3}} \\
& \left.+\frac{3}{D-3} u \cdot \nabla u_{A}-\frac{3}{D-3} u^{B} K_{B A}\right] \mathcal{P}_{C}^{A}=0
\end{aligned}
$$

\subsubsection{Divergence of velocity from a scalar constraint}

As we have explained in the previous section, the Einstein constraint equation $E^{S_{1}}$ is satisfied at all $R$ if it is satisfied at $R=0$. As explained in the previous subsection, the equation at $R=0$ simply asserts that

$$
\nabla \cdot u_{2}=-\frac{2(D-3)}{\mathcal{K}} \mathcal{V}^{S_{1}}(0)
$$

Reading off the value of $\mathcal{V}^{S_{1}}(0)$ from the table 7 we find

$$
\nabla \cdot u=\frac{(\nabla \cdot u)_{2}}{D-3}=\frac{1}{2 \mathcal{K}}\left(\nabla_{(A} u_{B)} \nabla_{(C} u_{D)} \mathcal{P}^{B C} \mathcal{P}^{A D}\right)
$$




\subsection{Second order corrections to the metric}

\subsubsection{Tensor sector}

The metric correction in the tensor sector is given by (3.3)

$$
\begin{aligned}
H_{A B}^{(T)} & =\left(\frac{-2(D-3)^{2}}{\mathcal{K}^{2}}\right) \int_{R}^{\infty} \frac{d y}{e^{y}-1} \int_{0}^{y} e^{x} \mathcal{S}_{A B}^{T}(x) d x \\
& =\left(\frac{2(D-3)^{2}}{\mathcal{K}^{2}}\right)\left[\log \left(1-e^{-R}\right) \int_{0}^{R} e^{x} S_{A B}^{T}(x) d x+\int_{R}^{\infty} \log \left(1-e^{-x}\right) e^{x} S_{A B}^{T}(x)\right]
\end{aligned}
$$

where $\mathcal{S}_{A B}^{T}$ is the second order source listed in table 6 . All the integrals that appear in the final answer can easily be performed analytically, but the final results (given in terms of polylogs) are not very illuminating; we prefer to leave our final result in terms of an explicit integral.

\subsubsection{Vector sector}

The solution for $H_{M}^{(V)}(R)$ at second order is given by (3.14)

$$
H_{M}^{(V)}(R)=\left(\frac{2(D-3)^{2}}{\mathcal{K}^{2}}\right)\left(e^{-R} \int_{0}^{R}\left(\frac{-e^{x}}{1-e^{-x}}\right) \mathcal{S}_{M}^{V 2}(x) d x-\int_{R}^{\infty} \frac{\mathcal{S}_{M}^{V 2}(x)}{1-e^{-x}}+e^{-R} \int_{0}^{\infty} \frac{\mathcal{S}_{M}^{V 2}(x)}{1-e^{-x}}\right)
$$

with all sources read off at 2nd order from table 6 . As in the tensor sector, all integrals that appear in (4.14) can be explicitly performed in terms of polylogs, but we find the expression (4.14) in terms of explicit integrals more illuminating.

\subsubsection{Scalar sector}

Equation $R^{S_{1}}$ is decoupled equation for $H^{(T r)}$. The integrated form is given by $(3.20)$ which we write again

$$
\begin{aligned}
H^{(T r)} & =\left(\frac{2(D-3)^{2}}{\mathcal{K}^{2}}\right) \int_{R}^{\infty} d y \int_{y}^{\infty} d x \mathcal{S}^{S_{1}}(x) \\
& =\left(\frac{2(D-3)^{2}}{\mathcal{K}^{2}}\right)\left[-R \int_{R}^{\infty} d x \mathcal{S}^{S_{1}}(x)+\int_{R}^{\infty} d x x \mathcal{S}^{S_{1}}(x)\right]
\end{aligned}
$$

The source $\mathcal{S}^{S_{1}}$ for 2 nd order is given in table 6 . Substituting this we get the final form of the metric correction

$$
H^{(T r, 2)}=-\left(\frac{2(D-3)^{2}}{\mathcal{K}^{2}}\right) e^{-R}(1+R)((u \cdot K-u \cdot \nabla u) \cdot \mathcal{P} \cdot(u \cdot K-u \cdot \nabla u))
$$

In a similar manner the fluctuation $H^{S}$ can is given by (3.25) upon plugging in the explicit values of the second order sources from tables 6,7 . 


\section{The spectrum of small fluctuations around a spherical membrane}

The simplest solution of the second order membrane equations of motion is a static spherical membrane dual to a Schwarzschild Black hole. In this section we compute the spectrum of small fluctuations about this solution. Our answers agree perfectly with earlier results for the spectrum of light quasinormal modes obtained by direct gravitational analysis, in [6]. We regard this detailed agreement as a nontrivial consistency check of the second order membrane equations of motion derived in this paper.

The computation presented in this section is a straightforward extension of the first order computation presented in section 5 of [9]. We have kept the discussion of this section brief. We refer the reader to section 5 of [9] for a fuller discussion of the logic behind our computation.

We work in standard spherical polar coordinates (see eq. 5.1 of [9]). The static spherical membrane is given by

$$
r=1, \quad u=-d t
$$

We study the small fluctuations

$$
\begin{aligned}
& r=1+\epsilon \delta r(t, \theta), \\
& u=-d t+\epsilon \delta u_{\mu}(t, \theta) d x^{\mu} .
\end{aligned}
$$

about this solution and work to linear order in $\epsilon$. As explained in [9], to linear order the metric on membrane worldvolume is given by

$$
d s^{2}=-d t^{2}+(1+2 \epsilon \delta r) d \Omega_{D-2}^{2} .
$$

As in [9] we find it convenient to work with covariant derivatives with respect to the unperturbed spherical metric

$$
d s^{2}=-d t^{2}+d \Omega_{D-2}^{2}
$$

The derivatives appearing from now on are all with respect to metric (5.4). We use the following notation for the laplacian with respect to this fixed metric

$$
\bar{\nabla}^{2}=\nabla_{\mu} \nabla^{\mu}=-\partial_{t}^{2}+\nabla_{a} \nabla^{a}=-\partial_{t}^{2}+\nabla^{2}
$$

\subsection{The divergence condition}

The r.h.s. of (1.5) is quadratic in $\epsilon$, and so vanishes upon linearizing in $\epsilon$. At linear order, therefore, (1.5) reduces to $\nabla \cdot u=0$ (where the divergence is taken along the dynamical membrane world volume). As explained in [9], this equation can be rewritten as

$$
\nabla_{\mu} \delta u^{\mu}=-(D-2) \partial_{t} \delta r
$$

where, the covariant derivatives (5.5) are now taken w.r.t. the fixed metric (5.4). $u^{0}$ deviates from unity only at quadratic order in $\epsilon$. For the linearized considerations of this section, therefore, the 1.h.s. of (5.5) is simply the spatial divergence of the velocity

$$
\nabla_{a} \delta u^{a}=-(D-2) \partial_{t} \delta r
$$


As in [9], (5.6) may be solved by separating $u$ into its gradient and curl parts, i.e. by setting

$$
\delta u_{a}=\nabla_{a} \Phi+\delta v_{a},
$$

with

$$
\nabla \cdot \delta v=0 .
$$

It follows from (5.6) that

$$
\nabla^{2} \Phi=-(D-2) \partial_{t} \delta r .
$$

\subsection{Linearized equation of motion}

In order to obtain the linearized membrane equations of motion we use eq. 5.7 of [9] together with

$$
\begin{aligned}
\frac{u^{E} K_{E B} K_{a}^{B}}{\mathcal{K}} & =-\epsilon \frac{\left(\nabla_{a} \partial_{t} \delta r-\delta u_{a}\right)}{D-2} \\
\frac{\nabla^{2} \nabla^{2} u_{a}}{\mathcal{K}^{3}} & =\epsilon \frac{\bar{\nabla}^{2} \bar{\nabla}^{2} \delta u_{a}+\bar{\nabla}^{2} \nabla_{a} \partial_{t} \delta r}{(D-2)^{3}} \\
\frac{K^{C D} \nabla_{C} \nabla_{D} u_{a}}{\mathcal{K}^{2}} & =\epsilon \frac{\bar{\nabla}^{2} \delta u_{a}-\nabla_{a} \partial_{t} \delta r}{(D-3)(D-2)} \\
\frac{\nabla_{a} \nabla^{2} \mathcal{K}}{\mathcal{K}^{3}} & =-\epsilon \frac{\nabla_{a} \bar{\nabla}^{2}\left(\bar{\nabla}^{2} \delta r+\delta r(D-2)\right)}{(D-2)^{3}} \\
\frac{\nabla_{a}\left(K^{B C} K_{B C} \mathcal{K}\right)}{\mathcal{K}^{3}} & =\epsilon \frac{3 \nabla_{a}\left(-\bar{\nabla}^{2} \delta r-\delta r(D-2)\right)}{(D-3)(D-2)}
\end{aligned}
$$

(the equations above are accurate only to linear order in $\epsilon$ and all covariant derivatives are taken with respect to (5.4)). The linearized membrane equation is given by

$$
\begin{aligned}
& {\left[\left(1+\frac{\bar{\nabla}^{2}}{D-2}\right) \delta u_{a}+\nabla_{a}\left(1+\frac{\bar{\nabla}^{2}}{D-2}\right) \delta r-\partial_{t} \nabla_{a} \delta r\left(1-\frac{1}{D-2}\right)-\partial_{t} \delta u_{a}\right]} \\
& +\left[\frac{\nabla_{a} \partial_{t} \delta r-\delta u_{a}}{D-2}+\frac{\bar{\nabla}^{2} \bar{\nabla}^{2} \delta u_{a}+\bar{\nabla}^{2} \nabla_{a} \partial_{t} \delta r}{(D-2)^{3}}+2 \frac{-\bar{\nabla}^{2} \delta u_{a}+\nabla_{a} \partial_{t} \delta r}{(D-3)(D-2)}+\frac{\nabla_{a} \bar{\nabla}^{2}\left(\bar{\nabla}^{2} \delta r+(D-2) \delta r\right)}{(D-2)^{3}}\right. \\
& \left.+3 \frac{\nabla_{a}\left(-\bar{\nabla}^{2} \delta r-(D-2) \delta r\right)}{(D-3)(D-2)}+3 \frac{\partial_{t} \delta u_{a}}{(D-3)}+3 \frac{\partial_{t} \nabla_{a} \delta r-\delta u_{a}}{(D-3)}\right]=0 .
\end{aligned}
$$

((5.10) generalizes equation (5.9) of [9]). Substituting (5.7) into (5.10) we find the generalized version of of (5.15) of [9],

$$
\begin{aligned}
& \left(\frac{\nabla^{2}}{D-2}+1-\partial_{t}+\frac{\bar{\nabla}^{2} \bar{\nabla}^{2}}{(D-2)^{3}}-\frac{2\left(\nabla^{2}\right)}{(D-2)^{2}}+\frac{3 \partial_{t}}{(D-3)}-\frac{3}{(D-3)}\right) \delta v_{a}= \\
& -\left(\frac{\partial_{t} \nabla_{a}}{D-2}+\frac{\nabla_{a} \nabla^{2}}{D-2}+\nabla_{a}-\nabla_{a} \partial_{t}+\frac{2 \nabla_{a} \partial_{t}}{(D-2)^{2}}-\frac{\nabla_{a} \bar{\nabla}^{2}\left(\nabla^{2}+(D-2)\right)}{(D-2)^{3}}\right. \\
& \left.-\frac{9 \nabla_{a}\left((D-2)^{2}-(D-2)\left(9 \nabla^{2}-\partial_{t}^{2}\right)\right)}{3(D-2)^{3}}+\frac{3 \partial_{t} \nabla_{a}}{(D-3)}\right) \delta r \\
& -\left(\frac{\nabla^{2}}{D-2}+1-\partial_{t}+\frac{\bar{\nabla}^{2} \bar{\nabla}^{2}}{(D-2)^{3}}-\frac{2\left(\nabla^{2}\right)}{(D-2)^{2}}+\frac{3 \partial_{t}}{(D-3)}-\frac{3}{(D-3)}\right) \nabla_{a} \Phi
\end{aligned}
$$




\subsection{Scalar quasinormal modes}

Using (5.6) and (5.9) we take the divergence of (5.11) to obtain

$$
\begin{aligned}
& -\left(\bar{\nabla}^{2}+D-3\right) \partial_{t} \delta r+\frac{\partial_{t} \nabla^{2} \delta r}{D-2}+\frac{\nabla^{2} \bar{\nabla}^{2} \delta r}{(D-2)}+\nabla^{2} \delta r-\partial_{t} \nabla^{2} \delta r-(D-2) \partial_{t} \delta r+(D-2) \partial_{t}^{2} \delta r \\
& +\frac{\nabla^{2} \partial_{t} \delta r+(D-2) \partial_{t} \delta r}{D-2}-\frac{\left(\bar{\nabla}^{2}+D-3\right)^{2}(D-2) \partial_{t} \delta r+\left(\bar{\nabla}^{2}+D-3\right) \nabla^{2} \partial_{t} \delta r}{(D-2)^{3}} \\
& +2 \frac{\left(\bar{\nabla}^{2}+D-3\right)(D-2) \partial_{t} \delta r+\nabla^{2} \partial_{t} \delta r}{(D-2)^{2}}+\frac{\nabla^{2} \bar{\nabla}^{2}\left(\bar{\nabla}^{2} \delta r+\delta r(D-2)\right)}{(D-2)^{3}} \\
& -\frac{\nabla^{2}\left(3 \nabla^{2} \delta r-\partial_{t}^{2} \delta r+3 \delta r(D-2)\right)}{(D-2)^{2}}-3 \frac{D-2}{(D-3)} \partial_{t}^{2} \delta r+\frac{3}{(D-3)}\left(\partial_{t} \nabla^{2} \delta r+(D-2) \partial_{t} \delta r\right)=0
\end{aligned}
$$

As in [9] we expand

$$
\delta r=\sum_{l, m} a_{l m} Y_{l m} e^{-i \omega_{l}^{r} t} .
$$

where the spherical harmonics $Y_{l m}$ obey

$$
-\nabla_{S^{D-2}}^{2} Y_{l m}=l(D+l-3) Y_{l m} .
$$

Inserting (5.13) into (5.12) we obtain

$$
\omega_{l}^{r}= \pm \sqrt{l-1}-i(l-1)+\frac{1}{D}\left( \pm \sqrt{l-1}\left(\frac{3 l}{2}-2\right)-i(l-1)(l-2)\right)
$$

The result (5.15) is in perfect agreement with the result obtained by EST in Equations (5.30) and (5.31) of [6].

As explained in [9], the modes with $l=0$ and $l=1$ are special. At $l=0$ the formula (5.15) yields $\omega=0,2 i-\frac{4 i}{D}$. The second solution is, however, spurious (see [9]). The first solution is the zero mode corresponding to rescaling the black hole; this is an exact zero mode at all orders in $1 / D$.

At $l=1$ (5.15) yields the frequencies $\omega=0,0$. As explained in [9] these two modes correspond to translations and boosts of the membrane.

\subsection{Vector quasinormal modes}

We expand the velocity fluctuations in a basis of vector spherical harmonic

$$
\delta v_{a}=\sum_{l, m} b_{l m} Y_{a}^{l m} e^{-i \omega_{l}^{v} t}
$$

Where, $l=1,2,3, \ldots$. The vector spherical harmonics satisfy the property

$$
\nabla^{2} V=-[(D+l-3) l-1] V
$$

Plugging (5.16) into (5.11), using (5.17) and equating the coefficients of independent vector spherical harmonics (see [9] for more discussion) we obtain

$$
\omega_{l}^{v}=-i(l-1)-\frac{i}{D}(l-1)^{2} .
$$


(5.18) is in perfect agreement with the formula (5.22) of [6] derived earlier by EST by purely gravitational analysis. Note that the mode with $l=1$ has vanishing frequency. As explained in [9] $l=1$ is the exact zero mode corresponding to setting the black hole spinning.

\section{Discussion}

In this paper we have worked out the duality between the dynamics of black holes in a large number of dimensions and the motion of a non gravitational membrane in flat space to second subleading order in $1 / D$. Our work generalizes the analysis of $[8,9]$. The concrete new results of this paper are

- The second order corrected membrane equations of motion listed in (1.4).

- The formula (1.5) for the divergence of the velocity field (which vanished at first order).

- The explicit form of the second order corrected metric dual to any given membrane motion (see subsection 4.2

In addition to obtaining the new results listed above we have also achieved an improved understanding of the structure of the perturbative expansion in $1 / D$. We have demonstrated that the perturbative programme, implemented to first nontrivial order in $[8,9]$, can systematically be extended to every order in the $1 / D$ expansion. In particular we have shown that the algebraically nontrivial 'integrability' properties that allowed for the existence of a first order solution in $[8,9]$ are actually automatic at all orders as as a consequence of the well known equation (2.22).

We have also explained that the membrane equations may directly be obtained by evaluating the Einstein constraint equation on the event horizon. In particular the membrane equations at $(n+1)^{\text {th }}$ order in $1 / D$ are obtained by evaluating the constraint equations on $n^{\text {th }}$ order metric, without needing to solve for the $(n+1)^{\text {th }}$ order metric. We have also explained that the assumption of $\mathrm{SO}(D-p-2)$ isometry, made in [9], is not necessary; the membrane equations can be derived under much more general conditions

The fact our membrane equations arise from the Einstein constraint equations at the event horizon is strongly reminiscent of the 'traditional' membrane paradigm of black hole physics. It would be very interesting to better understand the relationship between the the large $D$ membrane and the traditional membrane paradigm. [26-28].

As black holes are thermodynamical objects, the black hole membrane studied in $[8$, $9]$ and this paper should carry an entropy current. At leading order in $1 / D$ it turns out (see [29]) that this entropy current is given simply by a constant times $u^{M}$. The divergence of this entropy current is thus proportional to $\nabla . u$. It follows that the r.h.s. of the formula (1.5) gives an expression for the rate of entropy production on the membrane. It would be interesting to further investigate this observation and its consequences. 
On a related note, it would be interesting to derive the most general stationary solution of the second order corrected equations of motion derived in this paper and compare our results with those of [11].

In this paper we have focused our attention on black holes propagating in an otherwise perfectly flat spacetime. It would be interesting to generalize our study to the motion of black holes propagating in any vacuum solution of Einstein's equations, e.g. a gravity wave. Such a generalization would allow us, for instance, to study the absorption of gravity waves by black holes at large $D$. At first order in the derivative expansion we expect the generalized effective membrane equation to be given simply by covariantizing first order flat space equations of motion. At second order, however, the equations of motion could receive genuinely new contributions from the background Riemann tensor of the space in which the black hole propagates. ${ }^{16}$ It would be interesting to work this out in detail.

Finally, it would be interesting to put the membrane equations derived in this paper to practical use to allow us to learn new things about black holes. One possible direction would be to test out how well the large D expansion does in astrophysical contexts (i.e. when $D=$ 4). Another direction would be to use the formalism developed herein to address interesting unanswered structural questions about gravity, e.g. questions about the second law of thermodynamics in higher derivative gravity. We leave such investigations for the future.

\section{Acknowledgments}

We would like to thank K. Inbasekar, S. Thakur and M. Mandlik for many useful discussions during the progress in the project. We would like especially to thank S. Bhattacharyya for several very useful discussions and explanations. S. Mazumdar would like to acknowledge the hospitality of the University of Barcelona, ETH Zurich and IISER Mohali while this work was in progress. Y.D. and S. Mazumdar would also like to thank ICTP, Trieste for hospitality while this work was in progress. Y.D., A.D., S. Mazumdar and A.S would also like to thank the organizers of The Fourth Indo-Israel Meeting, Goa for hospitality while this work was in progress. S.M. would like to thank IAS Princeton for hospitality while this work was in progress. The work of all authors was supported by the Infosys Endowment for the study of the Quantum Structure of Spacetime, as well as an Indo Israel (UGC/ISF) grant. Finally we would all like to acknowledge our debt to the people of India for their steady and generous support to research in the basic sciences.

\section{A Method of calculation}

In this appendix we outline the method we have employed to obtain the results quoted in tables $2,3,4,5,6,7$.

As we have mentioned in the main text, our starting point is the metric listed in $(2.7),(2.8),(2.9),(2.10)$. In order to obtain the equations of motion listed in table 2 (see also table 3 ) we simply plugged this metric into the vacuum Einstein equations. Assuming these equations are already obeyed at $n-1$ order we then obtained the form of the $n^{\text {th }}$

\footnotetext{
${ }^{16}$ Something similar happens in the study of forced fluids in the fluid gravity correspondence [30].
} 
order equations. As emphasized in table 2, each of these equations have a 'homogeneous' contribution and a 'source' contribution. The homogeneous contribution is linear in the (as yet unknown) $n^{\text {th }}$ order fluctuation, and takes the same form at all orders. In order to evaluate the homogeneous contribution to all equations of motion, consequently, it is sufficient to work at first order.

While the first order computation is straightforward to perform analytically in principle, in practice the computations involved are rather lengthy. ${ }^{17}$ In order to guard against error we employed Mathematica in our computations using the following device. Following $[8,9]$ we specialized to the particular case of metrics that preserve an $\mathrm{SO}(D-p-2)$ isometry. Such special metrics effectively depend only on $p+3$ variables. For small values of $p$, therefore, all computations can be effectively performed on Mathematica (see [9] for a detailed explanation of how this is done). The first order computation performed in this manner yields the homogeneous part of the differential equations listed in tables 2 and 3 in a straightforward manner. Note that the homogeneous part of the equations are differential operators only in the variable $R$. They are 'ultra-local' on the membrane. Consequently, even though the assumption of isometry was used as a trick to facilitate the computation of the homogeneous part of the equation, the final result obtained for the structure of the equations listed in tables 2 and 3 is valid assuming only that all background quantities (e.g. $\mathcal{K})$ scale in the manner assumed in the text. In particular the homogeneous contribution to these equations are independent of $p$. By repeating all of our computations for $p=2$ and $p=3$ we have explicitly checked that this is the case.

Apart from the homogeneous pieces, the equations listed in tables 2 and 3 also have contributions from sources. Source terms are different at different orders in the computation. We obtained our explicit results for the first order sources listed in tables 4,5 and second order sources listed in tables 6,7 as follows. Working separately in the scalar, vector and tensor channels we first explicitly listed all possible source structures that could appear in any given equation both at first and second order in perturbation theory. The source structures that appear in our classification are the analogues of the 'geometrical' quantities listed in the 1.h.s. of table 4 in [9]. At any given order, it follows that the sources that appear in the equations of tables 2 and 3 are linear combinations of these structures with coefficients that are as yet unknown functions of $R$. We then worked out the analogue of the r.h.s. of table 4 of [9], i.e. we explicitly evaluated each of these basis source terms in terms of 'reduced source data' - the analogue of the expressions listed in table 1 of [9].

Using our explicit computations on Mathematica we read off the coefficients of all reduced sources in all of the equations listed in table 2 and 3. We then used our reduction formulae for 'geometrical sources in terms of reduced sources' (analogue of table 4 in [9]) to determine the coefficients of all source terms in the original geometrical basis of possible source terms. The last step (determination of geometrical sources from the known coefficients of reduced sources) is unambiguous provided the map between geometrical and reduced sources in invertible, i.e. provided there does not exist a nontrivial linear combination of geometrical sources that maps to zero when re expressed in terms of reduced

\footnotetext{
${ }^{17}$ These computations have, however, also been performed analytically in the upcoming paper [31].
} 


\begin{tabular}{|c|}
\hline Scalar sector \\
\hline $\begin{array}{c}\mathcal{S}^{S_{1}}(R)=e^{-R}(1-R)((u \cdot K-u \cdot \nabla u) \cdot \mathcal{P} \cdot(u \cdot K-u \cdot \nabla u)) \\
\mathcal{S}^{S_{2}}(R)=-\frac{1}{2} e^{-R}(R-2)\left(K_{M N} K_{P Q} P^{N P} P^{M Q}-\frac{\mathcal{K}^{2}}{D-3}\right)+\frac{1}{2} e^{-R}(R+2)\left(\nabla_{M} u_{N} \nabla_{P} u_{Q} P^{N P} P^{M Q}\right) \\
-\frac{e^{-R}}{2}\left(\nabla_{[M} u_{N]} \nabla_{[P} u_{Q]} P^{N P} P^{M Q}\right)-e^{-R} R\left(\nabla_{M} u_{N} K_{P Q} P^{N P} P^{M Q}\right) \\
+\frac{1}{\mathcal{K}} \frac{e^{-R}(R-2) R}{4} \nabla^{A}\left(\frac{D-3}{\mathcal{K}}\left(\frac{D-3}{\mathcal{K}^{3}}\left(\nabla \nabla^{2} \mathcal{K}-\nabla^{2} \nabla^{2} u\right)+8(u \cdot K-u \cdot \nabla u)+u \cdot K+\frac{\nabla^{2} u}{\mathcal{K}}\right)_{B} \mathcal{P}_{A}^{B}\right) \\
-\frac{e^{-R}(R-2) R}{4} \frac{\nabla^{2} \nabla^{2} \mathcal{K}}{\mathcal{K}^{3}}+\frac{1}{4} e^{-2 R}\left(e^{R}\left(R^{2}+2 R-4\right)-2(R-2) R\right)\left(u \cdot \nabla u_{M}\right)\left(u . \nabla u_{N}\right) \mathcal{P}^{M N} \\
+\frac{1}{2} e^{-2 R}\left(2 e^{R}(R-1)-(R-2) R\right)\left(u^{A} K_{A M}\right)\left(u^{B} K_{B N}\right) \mathcal{P}^{M N}+e^{-2 R}(R-2) R\left(u \cdot \nabla u_{M}\right)\left(u^{C} K_{C N}\right) P^{M N} \\
+\frac{1}{4} e^{-R}(R-2) R\left(\frac{\nabla^{2} u_{M}}{\mathcal{K}}\right)\left(\frac{\nabla^{2} u_{N}}{\mathcal{K}}\right) C P^{M N}-\frac{e^{-R}(R-2) R}{2}\left(\frac{\nabla^{2} u_{M}}{\mathcal{K}}\right)\left(u \cdot \nabla u_{N}\right) \mathcal{P}^{M N} \\
+\frac{1}{4} e^{-R} R\left(2 R^{2}-3 R-6\right) \frac{(u \cdot \nabla \mathcal{K})^{2}}{\mathcal{K}^{2}}-\frac{e^{-R}\left(R^{3}-14 R^{2}+20 R+4\right)}{4} u \cdot K \cdot u \frac{\mathcal{K}}{(D-3)} \\
+\frac{e^{-R}\left(3 R^{3}-38 R^{2}+62 R-4\right)}{4} \frac{\mathcal{K}}{(D-3)} \frac{u \cdot \nabla \mathcal{K}}{\mathcal{K}}-\frac{1}{4} e^{-R} R\left(R^{2}-6\right) u \cdot K \cdot u \frac{u \cdot \nabla \mathcal{K}}{\mathcal{K}}+e^{-R}(R-1) \frac{\mathcal{K}^{2}}{(D-3)^{2}} \\
\left.-\frac{1}{4} e^{-R}\left(\nabla_{(A} u_{B)} \nabla_{(C} u_{D}\right) \mathcal{P}^{B C} \mathcal{P}^{A D}\right) \\
\mathcal{S}^{S_{3}}(R)=\mathcal{V}^{S_{1}}(R)-\left(1-e^{-R}\right) \mathcal{S}^{S_{2}}(R) \\
\mathcal{S}^{S_{4}}(R)=\left(1-e^{-R}\right) \mathcal{S}^{S_{1}}(R)-2 \mathcal{V}^{S_{2}}(R)\end{array}$ \\
\hline Vector sector \\
\hline $\begin{array}{c}\mathcal{S}_{M}^{V_{1}}(R)=\frac{1}{\left(1-e^{-R}\right)}\left(\mathcal{V}_{L}^{V}(R)-\mathcal{S}_{L}^{V_{2}}(R)\right) \\
\mathcal{S}_{A}^{V_{2}}(R)=\frac{\mathcal{K}^{2}}{2(D-3)^{2}}\left[-e^{-2 R}\left(e^{R}-1\right)\left(R^{2}-2\right) \frac{3}{2} \frac{D-3}{\mathcal{K}}\left(1+2 \frac{u \cdot \nabla \mathcal{K}(D-3)}{\mathcal{K}^{2}}-\frac{u \cdot K \cdot u(D-3)}{\mathcal{K}}\right)(u \cdot \nabla u-u \cdot K)_{B}\right. \\
-e^{-2 R}\left(e^{R}-1\right)(R-1) \frac{(D-3)}{\mathcal{K}}\left(\frac{(D-3)}{\mathcal{K}^{3}}\left(\nabla \nabla^{2} \mathcal{K}-\nabla^{2} \nabla^{2} u\right)+8(u \cdot K-u \cdot \nabla u)+u \cdot K+\frac{\nabla^{2} u}{\mathcal{K}}\right)_{B} \\
\left.+R e^{-R}\left(-2 \frac{(D-3)^{2}}{\mathcal{K}^{2}}\left(\frac{\nabla_{M} \mathcal{K}}{\mathcal{K}}-u^{D} K_{D M}\right) P^{M N}\left(\nabla_{N} u_{B}-K_{N B}\right)+\frac{(D-3)}{\mathcal{K}}\left(u^{C} K_{C B}-\frac{\nabla^{2} u_{B}}{\mathcal{K}}\right)\right)\right] \mathcal{P}_{A}^{B} \\
-\frac{e^{-R}}{2} \frac{\mathcal{K}}{(D-3)}\left[-\mathcal{E}_{M}+D \frac{\nabla^{2} \nabla^{2} u_{M}}{\mathcal{K}^{3}}-D \frac{\nabla_{M}\left(\nabla^{2} \mathcal{K}\right)}{\mathcal{K}^{3}}+3 D \frac{(u \cdot K \cdot u)\left(u \cdot \nabla u_{M}\right)}{\mathcal{K}}-3 D \frac{(u \cdot K \cdot u)\left(u^{A} K_{A M}\right)}{\mathcal{K}}\right. \\
\left.-6 D \frac{(u \cdot \nabla \mathcal{K})(u \cdot \nabla u)}{\mathcal{K}^{2}}+6 D \frac{(u \cdot \nabla \mathcal{K})\left(u^{A} K_{A M}\right)}{\mathcal{K}^{2}}+3 u \cdot \nabla u-3 u^{A} K_{A M}\right] \mathcal{P}_{L}^{M}\end{array}$ \\
\hline Tensor sector \\
\hline $\begin{array}{c}\mathcal{S}_{L P}^{T}(R)=\left[e^{-R} \frac{\mathcal{K}}{(D-3)}\left(\left(K_{M N}-\nabla_{(M} u_{N)}\right)-\frac{\mathcal{P}_{M N}}{D}\left(K_{A B}-\nabla_{(A} u_{B}\right) \mathcal{P}^{A B}\right)\right. \\
-e^{-R}\left(\left(K_{M C}-\nabla_{C} u_{M}\right) \mathcal{P}^{C D}\left(K_{D N}-\nabla_{D} u_{N}\right)-\frac{\mathcal{P}_{M N}}{D}\left(K_{A C}-\nabla_{C} u_{A}\right) \mathcal{P}^{C D}\left(K_{D B}-\nabla_{D} u_{B}\right) \mathcal{P}^{A B}\right) \\
-\frac{1}{2} e^{-2 R}\left(R^{2}-4 R+2 e^{R}(R-1)+2\right)\left(\left(u_{C} K_{M}^{C}-u . \nabla u_{M}\right)\left(u_{C} K_{N}^{C}-u . \nabla u_{N}\right)\right. \\
\left.\left.-\frac{\mathcal{P}_{M N}}{D}\left(u_{C} K_{A}^{C}-u . \nabla u_{A}\right)\left(u_{C} K_{B}^{C}-u . \nabla u_{B}\right) \mathcal{P}^{A B}\right)\right] \mathcal{P}_{L}^{M} \mathcal{P}_{P}^{N}\end{array}$ \\
\hline
\end{tabular}

Table 6. Sources of $R_{M N}$ equations at 2 nd order.

sources (i.e. vanishes under the the assumption of isometry). We have verified that this condition is met at first order provided $p \geq 2$ and at second order provided that $p \geq 3 .{ }^{18}$ This is the reason we performed our computations at $p=3 .{ }^{19}$

\footnotetext{
${ }^{18} \mathrm{It}$ is easy to understand the inequalities listed here. When $p=1$, for instance, a potential source term proportional to the shear of the velocity field trivially vanishes just because fluids in one spatial dimension do not have a transverse direction in which to shear.

${ }^{19} \mathrm{We}$ also performed all computations in $p=2$ and verified that we obtained the same results for all sources from this computation - except in the case of a single second order source that vanished at $p=2$ but not at $p=3$. The coefficient of this term was left undetermined at $p=2$ but we determined at $p=3$.
} 


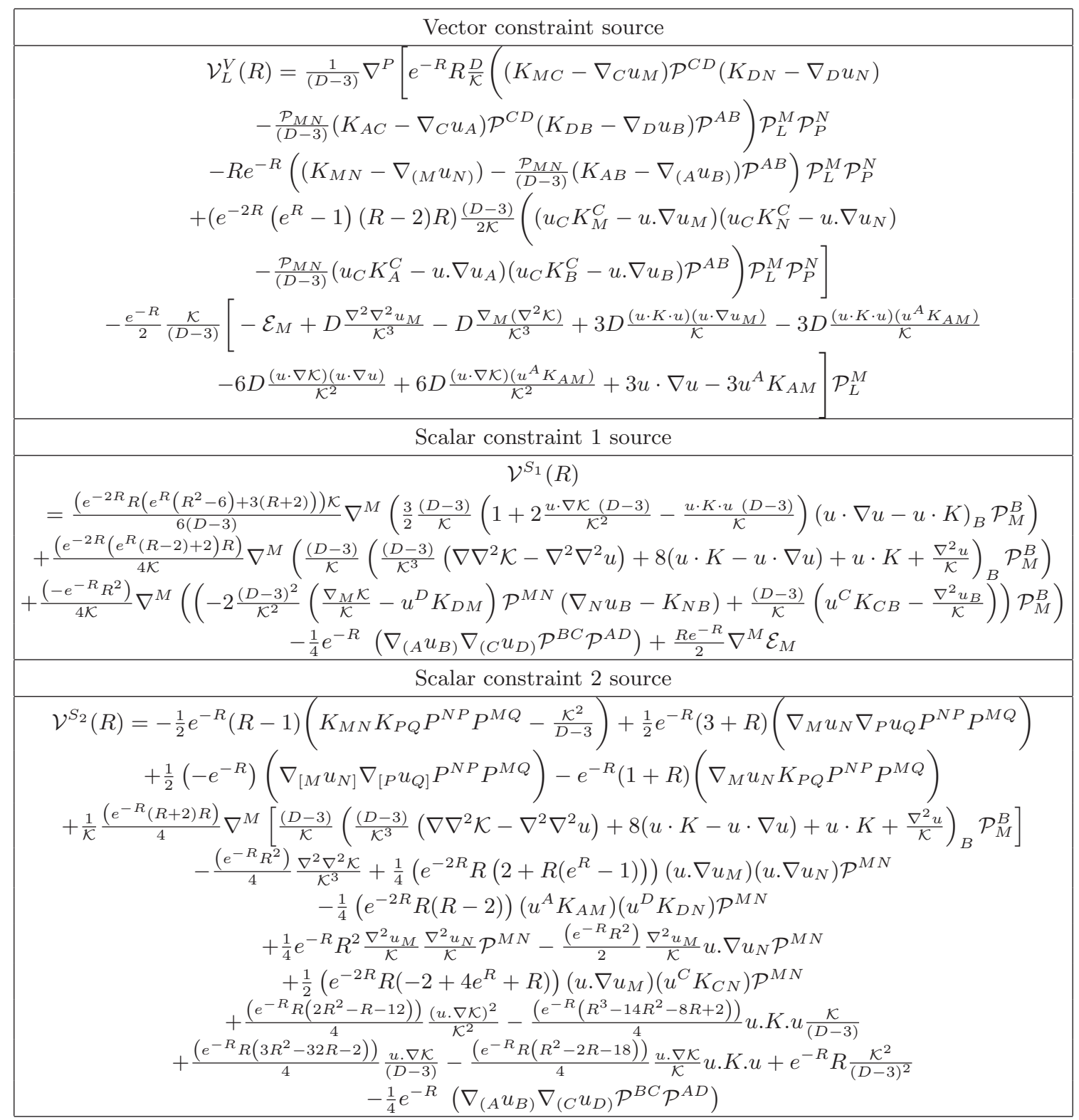

Table 7. Sources to constraint equations at 2 nd order.

\section{B Sources at second order}

In this appendix we present an explicit listing of all the sources that appear in the second order computation. By explicit computation we find that the sources listed in tables 1 and 2 are given at second order by the expressions we list in table 6 above.

Open Access. This article is distributed under the terms of the Creative Commons Attribution License (CC-BY 4.0), which permits any use, distribution and reproduction in any medium, provided the original author(s) and source are credited. 


\section{References}

[1] R. Emparan, R. Suzuki and K. Tanabe, The large D limit of general relativity, JHEP 06 (2013) 009 [arXiv: 1302.6382] [INSPIRE].

[2] R. Emparan, D. Grumiller and K. Tanabe, Large-D gravity and low-D strings, Phys. Rev. Lett. 110 (2013) 251102 [arXiv:1303.1995] [INSPIRE].

[3] R. Emparan and K. Tanabe, Holographic superconductivity in the large D expansion, JHEP 01 (2014) 145 [arXiv:1312.1108] [INSPIRE].

[4] R. Emparan and K. Tanabe, Universal quasinormal modes of large D black holes, Phys. Rev. D 89 (2014) 064028 [arXiv:1401.1957] [InSPIRE].

[5] R. Emparan, R. Suzuki and K. Tanabe, Instability of rotating black holes: large D analysis, JHEP 06 (2014) 106 [arXiv:1402.6215] [INSPIRE].

[6] R. Emparan, R. Suzuki and K. Tanabe, Decoupling and non-decoupling dynamics of large D black holes, JHEP 07 (2014) 113 [arXiv: 1406.1258] [INSPIRE].

[7] R. Emparan, R. Suzuki and K. Tanabe, Quasinormal modes of (anti-)de Sitter black holes in the $1 / D$ expansion, JHEP 04 (2015) 085 [arXiv: 1502.02820] [INSPIRE].

[8] S. Bhattacharyya, A. De, S. Minwalla, R. Mohan and A. Saha, A membrane paradigm at large D, JHEP 04 (2016) 076 [arXiv: 1504.06613] [INSPIRE].

[9] S. Bhattacharyya, M. Mandlik, S. Minwalla and S. Thakur, A charged membrane paradigm at large D, JHEP 04 (2016) 128 [arXiv: 1511.03432] [INSPIRE].

[10] R. Emparan, T. Shiromizu, R. Suzuki, K. Tanabe and T. Tanaka, Effective theory of black holes in the $1 / D$ expansion, JHEP 06 (2015) 159 [arXiv: 1504.06489] [INSPIRE].

[11] R. Suzuki and K. Tanabe, Stationary black holes: large D analysis, JHEP 09 (2015) 193 [arXiv: 1505.01282] [INSPIRE].

[12] K. Tanabe, Instability of the de Sitter Reissner-Nordström black hole in the $1 / D$ expansion, Class. Quant. Grav. 33 (2016) 125016 [arXiv:1511.06059] [INSPIRE].

[13] K. Tanabe, Charged rotating black holes at large D, arXiv:1605.08854 [INSPIRE].

[14] R. Emparan, R. Suzuki and K. Tanabe, Evolution and end point of the black string instability: large D solution, Phys. Rev. Lett. 115 (2015) 091102 [arXiv:1506.06772] [INSPIRE].

[15] R. Suzuki and K. Tanabe, Non-uniform black strings and the critical dimension in the $1 / D$ expansion, JHEP 10 (2015) 107 [arXiv:1506.01890] [INSPIRE].

[16] K. Tanabe, Black rings at large D, JHEP 02 (2016) 151 [arXiv:1510.02200] [InSPIRE].

[17] R. Emparan, K. Izumi, R. Luna, R. Suzuki and K. Tanabe, Hydro-elastic complementarity in black branes at large D, JHEP 06 (2016) 117 [arXiv:1602.05752] [INSPIRE].

[18] K. Tanabe, Elastic instability of black rings at large D, arXiv:1605.08116 [INSPIRE].

[19] A. Sadhu and V. Suneeta, Nonspherically symmetric black string perturbations in the large dimension limit, Phys. Rev. D 93 (2016) 124002 [arXiv:1604.00595] [INSPIRE].

[20] C.P. Herzog, M. Spillane and A. Yarom, The holographic dual of a Riemann problem in a large number of dimensions, JHEP 08 (2016) 120 [arXiv:1605.01404] [INSPIRE]. 
[21] M. Rozali and A. Vincart-Emard, On brane instabilities in the large D limit, JHEP 08 (2016) 166 [arXiv: 1607.01747] [INSPIRE].

[22] B. Chen, Z.-Y. Fan, P. Li and W. Ye, Quasinormal modes of Gauss-Bonnet black holes at large D, JHEP 01 (2016) 085 [arXiv: 1511.08706] [INSPIRE].

[23] G. Giribet, Large D limit of dimensionally continued gravity, Phys. Rev. D 87 (2013) 107504 [arXiv: 1303.1982] [INSPIRE].

[24] P. Dominis Prester, Small black holes in the large D limit, JHEP 06 (2013) 070 [arXiv: 1304.7288] [INSPIRE].

[25] B. Chen and P.-C. Li, Instability of charged Gauss-Bonnet black hole in de Sitter spacetime at large $D$, arXiv: 1607.04713 [INSPIRE].

[26] R.H. Price and K.S. Thorne, Membrane viewpoint on black holes: properties and evolution of the stretched horizon, Phys. Rev. D 33 (1986) 915 [InSPIRE].

[27] R.H. Price and K.S. Thorne, The membrane paradigm for black holes, Sci. Amer. 258 (1988) 69.

[28] T. Damour, Black hole eddy currents, Phys. Rev. D 18 (1978) 3598 [InSPIRE].

[29] S. Bhattacharyya et al., Currents, radiation and thermodynamics from the large D black hole membrane, to appear, (2015).

[30] S. Bhattacharyya et al., Forced fluid dynamics from gravity, JHEP 02 (2009) 018 [arXiv:0806.0006] [INSPIRE].

[31] S. Bhattacharyya et al., The membrane paradigm in arbitrary background, to appear, (2016). 